\title{
Adaptive reconstruction for azimuth signal of multichannel HRWS SAR imaging system
}

\author{
Linjian Zhang* ${ }^{*}$, Yesheng Gao and Xingzhao Liu
}

\begin{abstract}
The reconstruction of azimuth signal in multichannel synthetic aperture radar (SAR) for high-resolution and wide-swath (HRWS) imaging requires exact steering vectors. The information of ambiguities and system parameters are used to create the steering vectors. The knowledge of ambiguities involves ambiguity number and index; the system parameters include the pulse repetition frequency (PRF), platform velocity, and channel spacing. However, in some cases, the knowledge of ambiguities is Doppler-variant and there exist errors in the system parameters, which may degrade the reconstruction performance. In this work, an adaptive reconstruction method is developed. Firstly, we discuss the azimuth sampling of multichannel SAR and derive the equation of ambiguity index. We then utilize the azimuth cross-correlation to determine the aliasing number. Afterwards, based on the spatial spectrum estimation methods, an equivalent system parameter is adaptively calculated. With the aliasing number and the equivalent parameter, we can obtain the ambiguity information of each Doppler bin. Therefore, the steering vectors are constructed and the azimuth ambiguities can be suppressed. Compared with state-of-the-art reconstruction methods, our method achieves excellent performance even in highly nonuniform sampling case. Without relying on any system parameters, the proposed method has better practicality and applicability. We conduct extensive experiments including simulations and real data processing to verify the effectiveness and evaluate the performance of the proposed method.
\end{abstract}

Keywords: Azimuth signal reconstruction, Cross-correlation, High-resolution and wide-swath (HRWS), Multichannel synthetic aperture radar (SAR)

\section{Introduction}

Synthetic aperture radar (SAR) is an excellent remote sensor with good capability to provide all-weather and all-day observation of Earth's surface [1, 2]. In recent years, the high-resolution and wide-swath (HRWS) SAR imaging has gained intensive attention from the research community [3-12]. However, the high azimuth resolution and wide swath coverage contradict each other in the conventional single channel SAR [3, 4]. In the light of the Nyquist sampling theory, the finer the azimuth resolution, the larger the operational pulse repetition frequency (PRF) will be. Whereas, the wide-swath coverage

\footnotetext{
${ }^{*}$ Correspondence: zhlj_sjtu@sjtu.edu.cn

Department of Electronic Engineering, Shanghai Jiao Tong University, 800 Dongchuan Road, 200240 Shanghai China
}

requires a low PRF to avoid range ambiguities. Consequently, the azimuth multichannel SAR is utilized to solve this inherent contradiction and obtain HRWS SAR images [5]. In general, multiple uniformly spaced receiver channels along track are configured in the multichannel SAR as shown in Fig. 1. The single transmitter of the multichannel SAR emits a broad beam signal with a low operational PRF to illuminate wide area. The receiver channels simultaneously collect the echo signal to compensate the temporal sampling.

Because of the low operational PRF, the Doppler spectrum for each channel data is aliased. The unambiguous recovery of the Doppler spectrum is essential for the HRWS SAR imaging. On the basis of the oneto-one correspondence between the Doppler frequency and azimuth squint angle, the spectrum components 


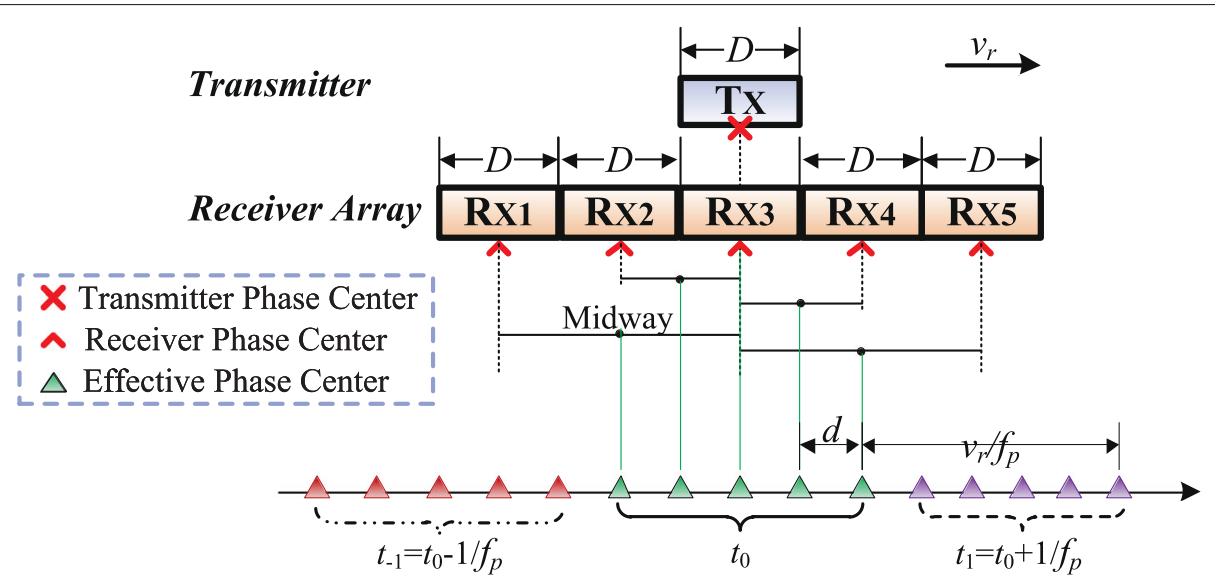

Fig. 1 Illustration of multichannel SAR. The positions of transmitter (Tx) and receivers (Rx), and corresponding positions of EPCs in azimuth multichannel SAR system. Note that $D=2 d$

within one Doppler bin can be identified as virtual signal sources from different known directions [4]. Therefore, the azimuth signal of multichannel SAR can be reconstructed by digital beamforming (DBF) techniques in range-Doppler domain. The Krieger DBF algorithm [3] and the post-Doppler space-time adaptive processing (STAP) method [4] are two representative DBF methods for azimuth signal reconstruction [13]. The principle of Krieger DBF algorithm is to solve a system of linear equations to reconstruct the Doppler spectrum. In comparison, the post-Doppler STAP method employs the Capon beamformer to suppress the azimuth ambiguities [14]. However, the nonuniform azimuth sampling will deteriorate the performance of the aforementioned DBF methods in terms of the signal-to-noise ratio (SNR). From another perspective, the azimuth sampling of multichannel HRWS SAR can be regarded as the recurrent nonuniform sampling. Inspired by the theory of recurrent nonuniform sampling derived by Eldar and Margolis in $[15,16]$, a filterbank reconstruction algorithm is proposed in [17].

The steering vectors related to different spectrum components of one Doppler bin are the crucial point of DBF techniques for ambiguity suppression. The knowledge of Doppler ambiguities (i.e., ambiguity number and index) and system parameters consisting of the operational PRF, platform velocity and spacing between two adjacent channels are utilized to construct the steering vectors. Nevertheless, the number and index of ambiguities are considered to be constant over the baseband Doppler frequencies in the above DBF methods, causing the degradation of SNR in the nonuniform sampling cases [18]. In contrary, the knowledge of ambiguities is Dopplervariant in some sampling cases, which also complicates the construction of steering vectors. Several improved DBF methods for ambiguity suppression are presented in
[18-20]. The key issues of these improved methods are the calculation of effective Doppler bandwidth (EDB) and the ambiguity-index screening (AIS) operation, by which the information of ambiguities can be obtained. The method proposed in [18], termed as IDBF, defines an equivalent sampling spacing for the computation of EDB and then utilizes the Doppler spectrum weighting. The IDBF overcomes the drawbacks of Krieger DBF algorithm, but at the cost of degradation of azimuth ambiguity-tosignal ratio (AASR) and azimuth resolution. Inspired by IDBF, a method named as ImpMMSE is proposed in [20]. The ImpMMSE uses a weighting process for the calculation of EDB and the minimum mean square error (MMSE) criterion to suppress azimuth ambiguities. Correspondingly, a remarkable improvement of AASR is realized. Besides, [19] calculates the baseband Doppler centroid and the EDB based on the Capon estimation method [14].

In the previous improved DBF reconstruction algorithms, the calculation of EDB and the AIS operation are critically dependent upon the system parameters. However, the system parameters may be inaccurate or unknown in some applications, which degrades the performance of ambiguity suppression [19, 21-23]. In fact, the real value of velocity is influenced by platform perturbation [23]. Additionally, the PRF and channel spacing may be lacking or influenced by measured errors $[19,21]$.

In this paper, we propose an adaptive azimuth reconstruction algorithm for multichannel SAR. The proposed method can adaptively obtain the information of ambiguities and estimate an equivalent parameter of PRF, platform velocity, and channel spacing. Firstly, concerning the uniformity of azimuth sampling, we propose to classify the azimuth sampling of multichannel SAR as uniformsampling, under-sampling, and over-sampling. Based on this novel classification, the aliasing number, the number of aliased copies of Doppler spectrum to be reconstructed, 
will be computed more simply. We can use the azimuth cross-correlation (cf. [24-26]) is utilized to determine the aliasing number. Besides, the calculation equation of ambiguity index for each Doppler bin is deduced. Notice that the estimation of EDB is essentially equivalent to the determination of the aliasing number. Afterwards, we calculate the equivalent parameter $F_{p}$ by means of spatial spectrum estimation methods which have been widely used in generic imaging problems [27-29]. If there exist redundant channels, the idea of subspace-based methods can be utilized [30,31]; otherwise, we use the Capon estimator $[14,21]$. Based on the aliasing number and the equivalent parameter (denoted as $F_{p}$ ), the ambiguity number and ambiguity indexes of each Doppler bin can be acquired. Thus, the accurate steering vectors can be adaptively constructed based on only the raw data, without using any system parameters. Consequently, our method can obtain good performance of ambiguity suppression even when the azimuth sampling is highly nonuniform.

The rest of this paper is organized as follows. Section 2 briefly introduces the signal model of multichannel SAR. Different cases of azimuth sampling in multichannel SAR are investigated in Section 3. Section 4 presents the detailed derivation of the aliasing number and the equivalent system parameter. Extensive experimental results on simulations and real data processing and discussions are given in Section 5. Section 6 concludes this paper.

Notation Vectors are denoted by lowercase boldface letters and matrices by uppercase boldface letters. The symbols $(\bullet)^{\mathrm{T}},(\bullet)^{*}$, and $(\bullet)^{\mathrm{H}}$ denote the transpose operation, conjugate operation, and Hermitian transpose operation, respectively. $\underset{\eta}{\mathbb{E}}\{\bullet\}$ represents the expectation operation over $\eta$. Besides, $\operatorname{diag}\{\bullet\}$ represents a diagonal matrix constructed by a vector.

\section{Signal model}

As illustrated in Fig. 1, a single transmitter (Tx) is combined with $M$ uniformly spaced receiver (Rx) channels along track in the multichannel HRWS SAR system. The spacing between adjacent receiver channels is $D$. In terms of the data acquisition mode, the multichannel SAR signal can be regarded as transmitted and received both from the effective phase centers (EPCs) after the EPC processing $[5,12]$. The EPC is in the middle position of the transmitter and its respective receiver. Therefore, the displacement from $m$ th $(m=1, \cdots, M)$ EPC to the first one can be denoted as $d_{m}=(m-1) \cdot d$, where $d=D / 2$. With the EPC processing, the relationship between the signal received by $m$ th channel and the first channel in the two-dimensional time domain can be expressed as

$$
\begin{aligned}
x_{m}(\tau, \eta) & =x_{1}\left(\tau, \eta+\eta_{m}\right), \\
& =x_{0}\left(\tau, \eta+\eta_{m}\right)
\end{aligned}
$$

where $\eta_{m}=d_{m} / v_{r} ; \tau$ and $\eta$ represents the range and azimuth time, respectively; $v_{r}$ is the platform velocity; $x_{m}(\tau, \eta)$ is the signal recorded by $m$ th channel. Note that $x_{1}(\tau, \eta)$ is generated by the multichannel SAR and its Doppler spectrum is aliased as a result of the low operational PRF. Let $x_{0}(\tau, \eta)$ denote the reference signal, which can be regarded as recorded by a single-channel SAR under the condition that the Nyquist sampling criterion is satisfied.

As a result of the low operational PRF represented by $f_{p}$, the Doppler spectrum of each channel data is aliased. Suppose that there exist $I$, denoting the ambiguity number, azimuth ambiguities at baseband Doppler frequency $f_{b}$, where $-f_{p} / 2 \leq f_{b}<f_{p} / 2$. Let $x_{m}\left(\tau, f_{b}\right)$ and $s\left(\tau, f_{b}+\right.$ $i \cdot f_{p}$ ) represent the received signal of $m$ th channel in range-Doppler domain and the equivalent unambiguous Doppler spectrum at frequency $f_{b}+i \cdot f_{p}$ from range gate $\tau$, respectively. $i$ refers to the ambiguity index ranging from $i_{\text {min }}$ to $i_{\text {max }}$. Notice that, sometimes the variables $i_{\text {min }}$ and $i_{\max }$ vary with $f_{b}$, and hence the ambiguity number calculated by (2) is Doppler-variant [19].

$$
I=i_{\text {max }}-i_{\text {min }}+1
$$

Besides, let $n_{m}\left(\tau, f_{b}\right)$ be the zero-mean white Gaussian noise recorded by $m$ th channel. Thus using vector notation, the multichannel SAR signal in range-Doppler domain can be:

$$
\boldsymbol{x}\left(\tau, f_{b}\right)=\sum_{i=i_{\min }}^{i_{\max }} \boldsymbol{a}_{i}\left(f_{b}\right) s\left(\tau, f_{b}+i \cdot f_{p}\right)+\boldsymbol{n}\left(\tau, f_{b}\right),
$$

The $M \times 1$ vectors $\boldsymbol{x}\left(\tau, f_{b}\right)$ and $\boldsymbol{n}\left(\tau, f_{b}\right)$ are the multichannel output and the noise vector, respectively, which can be written as

$$
\begin{aligned}
& \boldsymbol{x}\left(\tau, f_{b}\right)=\left[x_{1}\left(\tau, f_{b}\right), \cdots, x_{M}\left(\tau, f_{b}\right)\right]^{\mathrm{T}}, \\
& \boldsymbol{n}\left(\tau, f_{b}\right)=\left[n_{1}\left(\tau, f_{b}\right), \cdots, n_{M}\left(\tau, f_{b}\right)\right]^{\mathrm{T}} .
\end{aligned}
$$

The Doppler-variant steering vector $\boldsymbol{a}_{i}\left(f_{b}\right)$ corresponding to the ambiguity component $s\left(\tau, f_{b}+i \cdot f_{p}\right)$ is formulated as

$$
\boldsymbol{a}_{i}\left(f_{b}\right)=\left[e^{j 2 \pi\left(f_{b}+i \cdot f_{p}\right) \cdot d_{1} / v_{r}}, \ldots, e^{j 2 \pi\left(f_{b}+i \cdot f_{p}\right) \cdot d_{M} / v_{r}}\right]^{\mathrm{T}}
$$

For the azimuth signal reconstruction, the EPC spacing $d$, platform velocity $v_{r}$ and operational PRF $f_{p}$ are important parameters. These system parameters can be converted into an equivalent parameter $F_{p}$, which can be expressed as

$$
F_{p}=f_{p} \frac{d}{v_{r}}
$$


As a consequence, we can define another new parameter

$$
F_{b}=f_{b} \frac{d}{v_{r}}
$$

where $-F_{p} / 2 \leq F_{b}<F_{p} / 2$. Since the value of $d / v_{r}$ can be regarded as constant, we can use $F_{p}$ and $F_{b}$ to represent $f_{p}$ and $f_{b}$, respectively. As a result, the steering vector $\boldsymbol{a}_{i}\left(f_{b}\right)$ can be reformulated as

$$
\begin{aligned}
\boldsymbol{a}_{i}\left(f_{b}\right) & \triangleq \boldsymbol{a}_{i}\left(F_{b}\right) \\
& =\left[1, \cdots, e^{j 2 \pi\left(F_{b}+i \cdot F_{p}\right) \cdot(M-1)}\right]^{\mathrm{T}} .
\end{aligned}
$$

The steering vector $\boldsymbol{a}_{i}\left(F_{b}\right)$ is equivalent to $\boldsymbol{a}_{i}\left(f_{b}\right)$. Therefore, we can construct the steering vector $\boldsymbol{a}_{i}\left(f_{b}\right)$ by (9) as long as $F_{p}$ and the ambiguity indexes $i$ are known. Besides, the steering vector $\boldsymbol{a}_{i}\left(f_{b}\right)$ is independent of range delay. The reconstruction of azimuth signal is implemented bin by bin in range direction, which means the knowledge of range delay is unnecessary in the process of azimuth ambiguity suppression. Therefore, we can reconstruct the azimuth signal when $F_{p}$ and $i$ are obtained.

\section{Azimuth sampling of multichannel SAR}

This section analyzes the azimuth multichannel SAR sampling in terms of the uniformity of azimuth sampling. In specific, we describe the proposed classification of multichannel SAR sampling and derive the calculation equation of ambiguity index.

The fundamental idea behind the multichannel HRWS SAR is that the lack of temporal sampling points are compensated by the spatial sampling points. The optimum PRF, represented by $f_{p o}$, fulfills a uniform space-time sampling along the synthetic aperture and can be written as

$$
f_{p o}=\frac{v_{r}}{M \cdot d} \text {. }
$$

It can be seen that $f_{p o}$ is determined by the platform velocity, channel number, and channel spacing. Unfortunately, such a rigid selection of PRF cannot always be satisfied because of the timing diagram constraints in practical applications [5]. Any deviation from the optimum frequency $f_{p o}$ will result in nonuniform sampling in azimuth. Accordingly, different kinds of azimuth sampling schemes of multichannel SAR are generated with the change of $f_{p}$ as demonstrated in Fig. 2.

We use the uniformity of azimuth sampling $\kappa$ as defined in Eq. (11) to quantitatively analyze the effect of $f_{p}$ on the azimuth sampling.

$$
\kappa=\frac{M d}{v_{r} / f_{p}} .
$$

The uniformity of azimuth sampling has crucial effect on the suppression of azimuth ambiguities. Combining (10) and (11) yields a new formation of $f_{p}$ :

$$
f_{p}=\kappa \cdot f_{p o} .
$$

We observe a proportional relationship between the uniformity of azimuth sampling $\kappa$ and the operational PRF $f_{p}$.

According to the value of the uniformity of azimuth sampling, the azimuth sampling can be classified into three categories: $\kappa=1$ refers to the uniform-sampling (Fig. 2a); $\kappa>1$ indicates the over-sampling (Fig. 2b); $\kappa<1$ represents the under-sampling (Fig. 2c). The oversampling scheme is described that $d_{0}$, the displacement from $M$ th EPC to the first one in the next pulse, is smaller than $d$. If $d_{0}$ is larger than $d$, the sampling is undersampling. Notice that $d_{0}$ would reduce with the increase of $\kappa$. When the sampling position of the first channel coincides with that of $M$ th channel, $\kappa$ becomes $M /(M-1)$ and the received data are redundant [5]. For the sake of system complexity of the multichannel SAR, $\kappa$ is assumed not to be larger than $M /(M-1)$ in this paper.

The aliasing number denoted by $N$ is defined as the number of aliased copies of the Doppler spectrum within the scope of $\left[-f_{p} / 2, f_{p} / 2\right]$ and can be calculated by

$$
N=\frac{B_{r d}}{f_{p}}
$$

where $B_{r d}$ represents the reconstructed EDB of multichannel SAR by ambiguity suppression. It should be noted that the maximum value of aliasing number is $M$. As derived in $[18,19]$, the uniform-sampling, where $B_{r d}$ is $M f_{p o}$, achieves optimal performance on ambiguity suppression. In this work, $B_{r d}$ is designed to approach $M f_{p o}$. Therefore, in the cases of uniform-sampling and undersampling where $\kappa \leq 1$, the aliasing number is $M$ and the value of $B_{r d}$ can be $\kappa \cdot M f_{p o}$ based on (12). Nevertheless, the aliasing number is less than $M$ in the case of over-sampling. When the case of coinciding-sampling ( $\kappa=M /(M-1)$ ) occurs, the aliasing number is $M-1$ and $B_{r d}$ is equivalent to $M \cdot f_{p o}$. Thus, the reconstructed EDB $B_{r d}$ for uniform-sampling and coinciding-sampling can both achieve the value of $M \cdot f_{p o}$. We can conclude that the aliasing number $N$ ranges from $M-1$ to $M$ for sampling schemes with $M /(M-1)>\kappa>1$. Besides, $\kappa \cdot M f_{p o}$ should be at least larger than the $3 \mathrm{~dB}$-Doppler bandwidth of multichannel SAR in under-sampling cases. Thus, in terms of the valuing of $\kappa$, we have the criterion:

$$
\frac{B_{d}}{M \cdot f_{p o}}<\kappa \leq \frac{M}{M-1}
$$

where $B_{d}$ is the $3 \mathrm{~dB}$-Doppler bandwidth of multichannel SAR.

As known, except the system parameters, the reconstruction of azimuth signal involves another three parameters: the aliasing number $N$, the ambiguity number $I$, and the ambiguity index $i$. The aliasing number is an overall concept reflecting the relationship between $B_{r d}$ and $f_{p} . N$ 

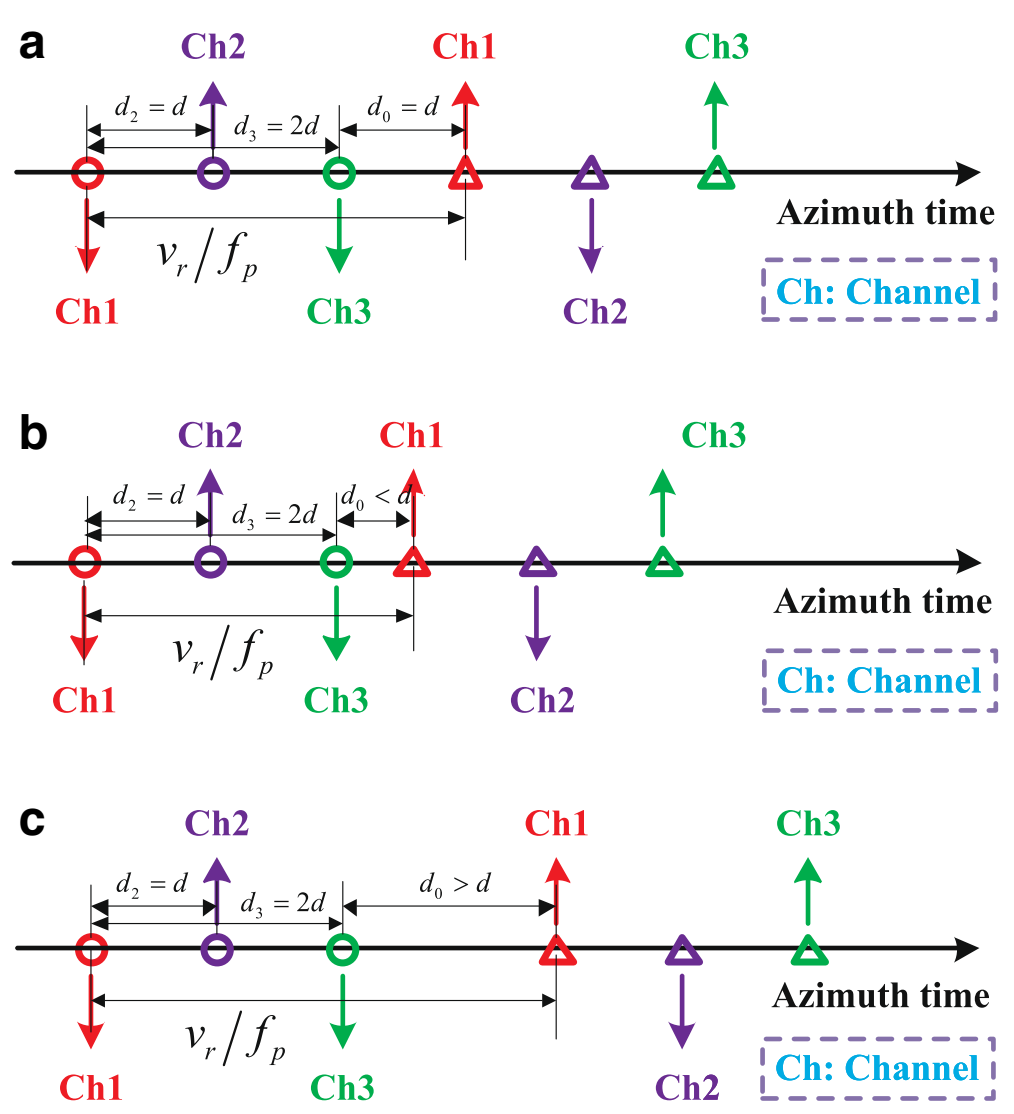

Fig. 2 Different azimuth sampling schemes for multichannel SAR regarding the positions of EPCs. a Uniform-sampling, $\kappa=1$. b Over-sampling, $\kappa>1$. $\mathbf{c}$ Under-sampling, $\kappa<1$. Here, the channel number is 3 , and $d_{1}=0$. Besides, the triangles and circles stand for the sampling positions in different pulses

may not be an integer in the over-sampling scheme. The azimuth ambiguities are just the spectrum components within a specific Doppler bin $f_{b}$. The number of azimuth ambiguities is referred to as ambiguity number, which must be an integer. Also, $I$ is Doppler-variant when the over-sampling scheme is generated. The ambiguity index $i$ indicates the connection between the instantaneous Doppler frequency $f_{a}$ [19] being reconstructed and the baseband Doppler frequency $f_{b}$, which can be expressed as

$$
f_{a}=f_{b}+i \cdot f_{p} \quad i \in\left[i_{\text {min }}, i_{\text {max }}\right] .
$$

Figure 3 shows the relational diagram between $f_{a}$ and $f_{b}$, which is the Doppler spectrum diagram (DSD) [19]. The ambiguity indexes $i$ may vary over $f_{b}$ under the influence of the channel number $M$ and the aliasing number $N$. When $M$ is odd, the index $i$ can be formulated by (16) (Fig. 3a); otherwise, $i$ can be acquired by (17) (Fig. 3b). Obviously, the ambiguity indexes $i$ are Doppler-variant in the case of over-sampling. With the equivalence between $f_{p}$ and $F_{p}$, we can determine the ambiguity indexes $i$ of each Doppler bin as long as $F_{p}$ and $N$ are obtained. We also observe that the ambiguity number $I$ is less than $M$ at some Doppler bins in the over-sampling scheme, meaning the existence of redundant channels. However, there is no redundant channel in the cases of uniform-sampling and under-sampling.

$i=\left\{\begin{array}{c}-\frac{M-1}{2}+1, \ldots, \frac{M-1}{2}, \quad-\frac{1}{2} f_{p} \leq f_{b}<\left(-\frac{1}{2}+\frac{M-N}{2}\right) f_{p} \\ -\frac{M-1}{2}, \ldots, \frac{M-1}{2}, \quad\left(-\frac{1}{2}+\frac{M-N}{2}\right) f_{p} \leq f_{b}<\left(\frac{1}{2}-\frac{M-N}{2}\right) f_{p} . \\ -\frac{M-1}{2}, \ldots, \frac{M-1}{2}-1, \quad\left(\frac{1}{2}-\frac{M-N}{2}\right) f_{p} \leq f_{b}<\frac{1}{2} f_{p}\end{array}\right.$

$$
i=\left\{\begin{array}{c}
-\frac{M}{2}+1, \ldots, \frac{M}{2}, \quad-\frac{1}{2} f_{p} \leq f_{b}<-\frac{M-N}{2} f_{p} \\
-\frac{M}{2}+1, \ldots, \frac{M}{2}-1, \quad-\frac{M-N}{2} f_{p} \leq f_{b}<\frac{M-N}{2} f_{p} \\
-\frac{M}{2}, \ldots, \frac{M}{2}-1, \quad \frac{M-N}{2} f_{p} \leq f_{b}<\frac{1}{2} f_{p}
\end{array}\right.
$$

\section{Adaptive reconstruction method}

In this section, we introduce the proposed adaptive azimuth reconstruction method for multichannel HRWS SAR. The proposed method can be separated into two principal procedures: the first one is the determination of the aliasing number $N$, and the other one is the estimation 


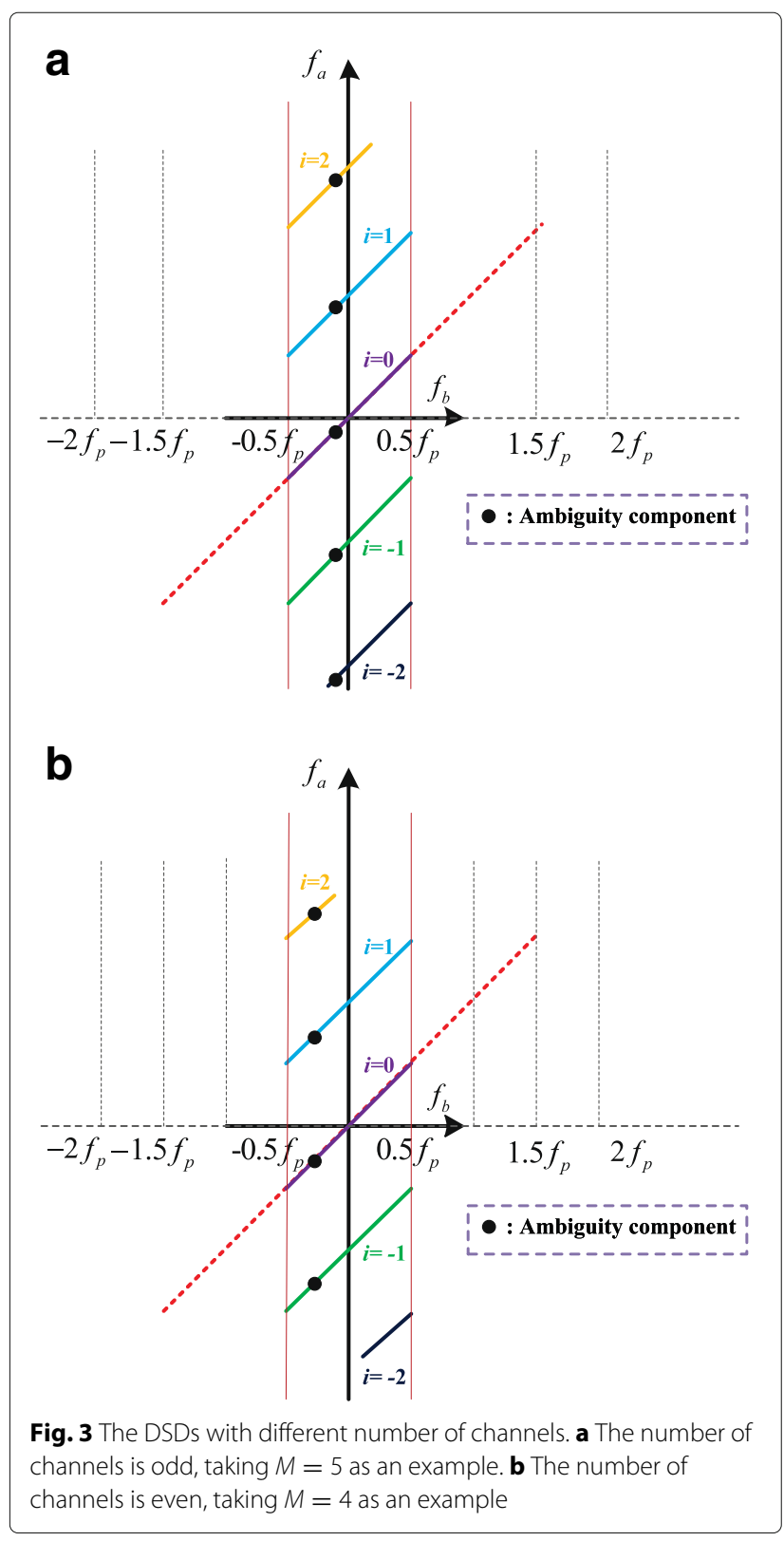

of the equivalent parameter $F_{p}$. Then the algorithm steps are given. Finally, we analyze the computation complexity of our adaptive reconstruction method, demonstrating low computation load.

\subsection{Determination of aliasing number}

Here we elaborate the determination of aliasing number $N$ by using cross-correlation technique presented in [26]. Based on the azimuth cross-correlation, the coherence between $x_{0}(\tau, \eta)$ and $x_{0}(\tau, \eta+\Delta \eta)$ can be

$$
\mu(\Delta \eta)=\frac{\underset{\eta}{\mathbb{E}}\left\{\left|x_{0}(\tau, \eta) x_{0}^{*}(\tau, \eta+\Delta \eta)\right|\right\}}{\sqrt{\underset{\eta}{\mathbb{E}}\left\{\left|x_{0}(\tau, \eta)\right|^{2}\right\} \underset{\eta}{\mathbb{E}}\left\{\left|x_{0}^{*}(\tau, \eta+\Delta \eta)\right|^{2}\right\}}},
$$

According to (1), the received data of $m$ th channel is just the delayed version of the first channel with time shift $\eta_{m}=d_{m} / v_{r}$ during a pulse. While, $\eta_{0}$, termed as the time shift between $M$ th channel in the former pulse and the first channel in the next pulse, is $\eta_{0}=d_{0} / v_{r}$. Recall that $d_{0}$ denotes the distance from the $M$ th EPC during the former pulse to the first EPC in the latter pulse. Thus, the time shift for adjacent azimuth samples generated from the same pulse is $\eta_{c}=d / v_{r}$; the time shift for adjacent azimuth samples in different pulses is $\eta_{0}$. Note that we derive that in the case of over-sampling, the expression $\eta_{0}<\eta_{c}$ can be obtained; otherwise, the expression $\eta_{0} \geq \eta_{c}$ can be determined.

Utilizing (1) and (18), the coherence of neighboring azimuth samples in the same pulse, denoted by $\alpha$, can be defined as

$$
\alpha=\frac{1}{M-1} \sum_{m=2}^{M} \frac{\underset{\eta}{\mathbb{E}}\left\{\left|x_{m-1}(\tau, \eta) x_{m}^{*}(\tau, \eta)\right|\right\}}{\sqrt{\frac{\mathbb{Z}}{\mathbb{E}}\left\{\left|x_{m-1}(\tau, \eta)\right|^{2}\right\} \underset{\eta}{\mathbb{E}}\left\{\left|x_{m}(\tau, \eta)\right|^{2}\right\}}},
$$

The coherence of adjacent samples from different pulses, namely $\gamma$, can be derived as

$$
\gamma=\frac{\underset{\eta}{\mathbb{E}}\left\{\left|x_{M}(\tau, \eta) x_{1}^{*}\left(\tau, \eta+1 / f_{p}\right)\right|\right\}}{\sqrt{\frac{\mathbb{Z}}{\mathbb{E}}\left\{\left|x_{M}(\tau, \eta)\right|^{2}\right\} \underset{\eta}{\mathbb{E}}\left\{\left|x_{1}\left(\tau, \eta+1 / f_{p}\right)\right|^{2}\right\}}} .
$$

Combining (1), (18), (19), and (20), the approximation of $\alpha$ and $\gamma$ can be obtained:

$$
\begin{aligned}
& \alpha=\mu\left(\eta_{c}\right), \\
& \gamma=\mu\left(\eta_{0}\right) .
\end{aligned}
$$

Considering that $\mu(\Delta \eta)$ decreases with an increasing $\Delta \eta$ [32], the azimuth sampling of multichannel SAR is oversampling when $\alpha<\gamma$; otherwise, the azimuth sampling is uniform-sampling or under-sampling. Furthermore, if the value of $\gamma$ approaches 1 , we can infer that $\eta_{0}$ is zero and the azimuth sampling is coinciding-sampling.

As discussed in Section 3, the aliasing number $N$ is influenced by the uniformity of azimuth sampling $\kappa$, which determines the azimuth sampling scheme. The value of $N$ can be expressed as:

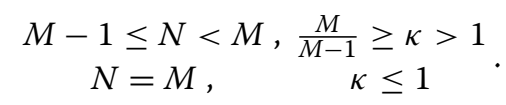

Bear in mind, in the case of uniform-sampling $(\kappa=1)$, the aliasing number $N$ equals $M$; in the coinciding-sampling case $(\kappa=M /(M-1)), N$ is just $M-1$. Hence, after the classification of azimuth sampling schemes, the aliasing number can be adaptively determined. With the description in (23), we only focus on the calculation of $N$ for the 
over-sampling scheme. Here, we formally present the criterion in terms of the determination of $N$ when $\kappa>1$. Based on the above analysis, we creatively propose the following determination criterion:

$$
\begin{aligned}
N & =\frac{\gamma-\alpha}{1-\alpha}(M-1)+\frac{1-\gamma}{1-\alpha} M \\
& =M-\frac{\gamma-\alpha}{1-\alpha}
\end{aligned} .
$$

Since $\alpha<\gamma \leq 1$ in the over-sampling scheme, (24) is a convex function related to the coherence of adjacent azimuth samples in multichannel SAR.

According to (24), $N$ is in the range from $M-1$ to $M$. For the azimuth uniform-sampling, $\gamma$ is equivalent to $\alpha$ and hence the aliasing number is $M$. When the operational PRF leads to coinciding-sampling, $\gamma$ reaches 1 and thus $N$ reduces to $M-1$. For other over-sampling schemes where $\kappa \in(1, M /(M-1)), N$ is larger than $M-1$ but less than $M$. Besides, as the increase of $\kappa$, the aliasing number reduces. Thus, we can conclude that the reconstructed EDB $B_{r d}$ approaches $M f_{p o}$ under the criterion formula expressed in (24).

With the coherence coefficients $\alpha$ and $\gamma$, we can clarify which case the azimuth sampling is. After that, the aliasing number $N$ can be directly obtained as $M$ or calculated by (24). Furthermore, we can adaptively decide the aliasing number without using any system parameter.

It should be noted that the coherence coefficients $\alpha$ and $\gamma$ are not obtained in reality due to the expectation operations in (19) and (20). $\alpha$ and $\gamma$ can be estimated by (25) and (26), respectively.

$$
\begin{aligned}
& \hat{\alpha}=\frac{1}{M-1} \sum_{m=2}^{M} \frac{\sum_{\eta}\left|x_{m-1}(\tau, \eta) x_{m}^{*}(\tau, \eta)\right|}{\sum_{\eta} \sqrt{\left|x_{m-1}(\tau, \eta)^{2}\right| \cdot\left|x_{m}(\tau, \eta)^{2}\right|}} \\
& \hat{\gamma}=\frac{\sum_{\eta}\left|x_{M}(\tau, \eta) x_{1}^{*}\left(\tau, \eta+1 / f_{p}\right)\right|}{\sum_{\eta} \sqrt{\left|x_{M}(\tau, \eta)^{2}\right| \cdot\left|x_{1}\left(\tau, \eta+1 / f_{p}\right)^{2}\right|}}
\end{aligned}
$$

For the purpose of improving estimation accuracy, we use all of the azimuth samples of each range gate to calculate coherence coefficients.

\subsection{Estimation of the equivalent system parameter}

In this part, we present the estimation of the equivalent system parameter $F_{p}$. The spatial spectrum estimation techniques can be utilized to adaptively estimate $F_{p}$ [33]. The principles of the classical algorithms, including Capon method, multiple signal classification (MUSIC) method and estimation of signal parameters via rotational invariance technique (ESPRIT), will be employed in this paper. Since there is no redundant channel in the uniformsampling or under-sampling case, only the Capon method can be used. In comparison, the three spectrum estimation techniques are all suitable for some Doppler bins of the over-sampling case. However, the subspace based methods have higher accuracy than Capon method. Also note that the Capon method and MUSIC method require spectral peak searching to obtain the estimation of $F_{p}$, which would complicate the estimation process. There should be a good balance between the estimation accuracy and the complexity regarding which technique is selected to calculate $F_{p}$.

\subsubsection{Capon method}

A new steering vector $\boldsymbol{b}\left(F_{a}\right)$ over the instantaneous Doppler frequency $f_{a}$ is defined as

$$
\begin{aligned}
\boldsymbol{b}\left(f_{a}\right) & \triangleq \boldsymbol{b}\left(F_{a}\right) \\
& =\left[1, e^{j 2 \pi F_{a}}, \cdots, e^{j 2 \pi(M-1) F_{a}}\right]^{\mathrm{T}},
\end{aligned}
$$

where $F_{a}$ is a new equivalent parameter expressed as

$$
F_{a}=f_{a} \cdot d / v_{r} \quad f_{a} \in\left[-\frac{M}{2} f_{p}, \frac{M}{2} f_{p}\right] .
$$

Combining (7) and (11) yields

$$
F_{p}=\frac{\kappa}{M} \text {. }
$$

Thus the inferior and superior limits of $F_{a}$ for estimating $F_{p}$ are $-\kappa / 2$ and $\kappa / 2$, respectively. Moreover, based on the categorization of the azimuth sampling scheme and the inequality (14), the scope of $F_{a}$ can be approximately determined.

The Capon spectrum at $F_{b}$ (equivalent to $f_{b}$ ) over $F_{a}$ is

$$
P_{\text {Capon }}\left(F_{a}, F_{b}\right)=\frac{1}{\boldsymbol{b}\left(F_{a}\right)^{\mathrm{H}} \boldsymbol{R}\left(F_{b}\right)^{-1} \boldsymbol{b}\left(F_{a}\right)},
$$

where $\boldsymbol{R}\left(F_{b}\right)$ is the covariance matrix of multichannel SAR signal at $F_{b} \cdot \boldsymbol{R}\left(F_{b}\right)$ can be expressed as

$$
\begin{aligned}
\boldsymbol{R}\left(F_{b}\right) & =\underset{\tau}{\mathbb{E}}\left[\boldsymbol{x}\left(\tau, f_{b}\right) \boldsymbol{x}^{\mathrm{H}}\left(\tau, f_{b}\right)\right] \\
& =\boldsymbol{A}\left(F_{b}\right) \boldsymbol{R}_{s s}\left(F_{b}\right) \boldsymbol{A}\left(F_{b}\right)^{\mathrm{H}}+\sigma_{n}^{2} \boldsymbol{I}_{M} .
\end{aligned}
$$

The array manifold $A\left(F_{b}\right)$ at $F_{b}$ is given by

$$
\boldsymbol{A}\left(F_{b}\right)=\left[\boldsymbol{a}_{i_{\text {min }}}\left(F_{b}\right), \cdots, \boldsymbol{a}_{i_{\text {max }}}\left(F_{b}\right)\right]^{\mathrm{T}},
$$

and the matrix $\boldsymbol{R}_{s s}\left(F_{b}\right)$ given by

$$
\boldsymbol{R}_{s s}\left(F_{b}\right)=\underset{\tau}{\mathbb{E}}\left[\boldsymbol{s}\left(\tau, f_{b}\right) \boldsymbol{s}\left(\tau, f_{b}\right)^{\mathrm{H}}\right],
$$

where $\boldsymbol{s}\left(\tau, f_{b}\right)=\left[s\left(\tau, f_{b}+i_{\min } f_{p}\right), \cdots, s\left(\tau, f_{b}+i_{\text {max }} f_{p}\right)\right]^{\mathrm{T}}$. Besides, $\sigma_{n}^{2}$ denotes the variance of noise, $\boldsymbol{I}_{M}$ is an identity matrix with size $M \times M$. Because of the unavailability of $\boldsymbol{R}\left(F_{b}\right)$ in practical applications, it is usually replaced with the sample covariance matrix $\hat{\boldsymbol{R}}\left(F_{b}\right)$ which is calculated by 


$$
\hat{\boldsymbol{R}}\left(F_{b}\right)=\frac{1}{L} \sum_{l=1}^{L}\left\{\boldsymbol{x}\left(\tau_{l}, f_{b}\right) \boldsymbol{x}^{\mathrm{H}}\left(\tau_{l}, f_{b}\right)\right\},
$$

where $L$ is the number of used training samples of every receiver channel and $\boldsymbol{x}\left(\tau_{l}, f_{b}\right)$ denotes the $l$ th training sample vector in range direction from $f_{b}$.

The peaks of $P_{\text {Capon }}\left(F_{a}, F_{b}\right)$ correspond to the ambiguities within Doppler bin $f_{b}$. Hence, the interval between adjacent peaks of the Capon spectrum $P_{\text {Capon }}\left(F_{a}, F_{b}\right)$ is just $F_{p}$.

\subsubsection{MUSIC method}

The eigen-decomposition of the covariance matrix $\boldsymbol{R}\left(F_{b}\right)$ as expressed in (31) can be

$$
\boldsymbol{R}\left(F_{b}\right)=\sum_{m=1}^{M} \lambda_{m} \boldsymbol{e}_{m} \boldsymbol{e}_{m}^{\mathrm{H}}=\boldsymbol{E}_{s} \boldsymbol{\Lambda}_{s} \boldsymbol{E}_{s}^{\mathrm{H}}+\boldsymbol{E}_{n} \boldsymbol{\Lambda}_{n} \boldsymbol{E}_{n}^{\mathrm{H}},
$$

where $\lambda_{1} \geq \lambda_{2} \geq \cdots \geq \lambda_{I} \geq \lambda_{I+1}=\cdots=$ $\lambda_{M}=\sigma_{n}^{2}$ are the eigenvalues of $\boldsymbol{R}\left(F_{b}\right)$ sorted in descending order and $\boldsymbol{e}_{m}$ are the corresponding eigenvectors. The eigenvalues and their corresponding eigenvectors are relevant to the baseband Doppler frequency $f_{b}$. The matrix $\boldsymbol{E}_{s}=\left[\boldsymbol{e}_{1}, \cdots \boldsymbol{e}_{I}\right]$ and matrix $\boldsymbol{E}_{n}=\left[\boldsymbol{e}_{I+1}, \cdots \boldsymbol{e}_{M}\right]$ are utilized to span the signal subspace and noise subspace, respectively. The formulations of matrices $\boldsymbol{\Lambda}_{s}$ and $\boldsymbol{\Lambda}_{n}$ are $\boldsymbol{\Lambda}_{s}=\operatorname{diag}\left\{\lambda_{1}, \cdots, \lambda_{I}\right\}$ and $\boldsymbol{\Lambda}_{s}=\sigma_{n}^{2} \boldsymbol{I}_{M-I}$, respectively. The dimension of the signal subspace is equivalent to the ambiguity number $I$ and can be obtained by using (2), (16) and (17). The MUSIC pseudo spatial spectrum at $F_{b}$ over $F_{a}$ can be calculated by

$$
P_{\text {MUSIC }}\left(F_{a}, F_{b}\right)=\frac{1}{\sum_{m=I+1}^{M}\left|\boldsymbol{e}_{m}^{\mathrm{H} b}\left(F_{a}\right)\right|^{2}} .
$$

The distance between the adjacent peaks in the pseudo spectrum $P_{\text {MUSIC }}\left(F_{a}, F_{b}\right)$ is just $F_{p}$.

\subsubsection{ESPRIT method}

Define two selection matrices with size $(M-1) \times M$ :

$$
\begin{aligned}
& \boldsymbol{J}_{1}=\left[\boldsymbol{I}_{M-1} \mid \mathbf{0}_{M-1}\right], \\
& \boldsymbol{J}_{2}=\left[\mathbf{0}_{M-1} \mid \boldsymbol{I}_{M-1}\right],
\end{aligned}
$$

where $\mathbf{0}_{M-1}$ indicates a zero vector with size $(M-1) \times 1$. Let us multiply the array manifold $\boldsymbol{A}\left(F_{b}\right)$ as in (32) by $\boldsymbol{J}_{1}$ and $\boldsymbol{J}_{2}$, respectively, two sub-matrices are obtained as

$$
\begin{aligned}
& \boldsymbol{A}_{1}\left(F_{b}\right)=\boldsymbol{J}_{1} \boldsymbol{A}\left(F_{b}\right), \\
& \boldsymbol{A}_{2}\left(F_{b}\right)=\boldsymbol{J}_{2} \boldsymbol{A}\left(F_{b}\right) .
\end{aligned}
$$

With the steering vector $\boldsymbol{a}_{i}\left(F_{b}\right)$ as formulated in (9), we can express the relationship between $\boldsymbol{A}_{1}\left(F_{b}\right)$ and $\boldsymbol{A}_{2}\left(F_{b}\right)$ as

$$
\boldsymbol{A}_{2}\left(F_{b}\right)=\boldsymbol{A}_{1}\left(F_{b}\right) \Psi
$$

where

$$
\boldsymbol{\Psi}=\operatorname{diag}\left\{e^{j 2 \pi \cdot i_{\min } \cdot F_{p}}, \cdots, e^{j 2 \pi \cdot i_{\max } \cdot F_{p}}\right\} .
$$

is just the translational invariance matrix induced by the configuration of uniformly spaced channels in the multichannel HRWS SAR.

Take into account that the variable $F_{b}$ on the right side of (35) is omitted for brevity. Then, multiplying matrix $\boldsymbol{E}_{s}\left(F_{b}\right)$ as appearing in (35) by $\boldsymbol{J}_{1}$ and $\boldsymbol{J}_{2}$, respectively, we have

$$
\begin{aligned}
& \boldsymbol{E}_{s 1}\left(F_{b}\right)=\boldsymbol{J}_{1} \boldsymbol{E}_{s}\left(F_{b}\right), \\
& \boldsymbol{E}_{s 2}\left(F_{b}\right)=\boldsymbol{J}_{2} \boldsymbol{E}_{s}\left(F_{b}\right) .
\end{aligned}
$$

According to the theory of ESPRIT [31], there exists an underlying rotational invariance between $\boldsymbol{E}_{s 1}\left(F_{b}\right)$ and $\boldsymbol{E}_{s 2}\left(F_{b}\right)$, which is expressed as

$$
\boldsymbol{E}_{s 2}\left(F_{b}\right)=\boldsymbol{E}_{s 1}\left(F_{b}\right) \boldsymbol{\Phi}\left(F_{b}\right) .
$$

$\boldsymbol{\Phi}\left(F_{b}\right)$ just represents the rotational invariance between signal subspaces. Moreover, $\boldsymbol{\Phi}\left(F_{b}\right)$ can be formulated as

$$
\boldsymbol{\Phi}\left(F_{b}\right)=\boldsymbol{T}\left(F_{b}\right)^{-1} \boldsymbol{\Psi} \boldsymbol{T}\left(F_{b}\right),
$$

where $\boldsymbol{T}\left(F_{b}\right)$ is a nonsingular matrix and can be written as

$$
\boldsymbol{T}\left(F_{b}\right)=\boldsymbol{R}_{s s}\left(F_{b}\right) \boldsymbol{A}^{\mathrm{H}}\left(F_{b}\right) \boldsymbol{E}_{s}\left(F_{b}\right)\left(\boldsymbol{\Lambda}_{s}-\sigma_{n}^{2} \boldsymbol{I}_{I}\right)^{-1} .
$$

From (46), we know that $\boldsymbol{\Psi}$ and $\boldsymbol{\Phi}\left(F_{b}\right)$ have the same eigenvalues; the diagonal elements of $\boldsymbol{\Psi}$ are just the eigenvalues of $\boldsymbol{\Phi}\left(F_{b}\right)$. The difference value between two adjacent phases (in radian) of the eigenvalues of $\boldsymbol{\Phi}\left(F_{b}\right)$ equals $2 \pi \cdot F_{p}$, and hence the value of $F_{p}$ can be acquired. Besides, $\boldsymbol{\Phi}\left(F_{b}\right)$ can be easily obtained by using $(45)$.

\subsection{Algorithm steps}

Based on the above derivation and discussion, here, we present the algorithm steps of the proposed adaptive azimuth reconstruction method as illustrated in Fig. 4. The implementation details are as follows:

Step 1: Perform azimuth cross-correlation to the multichannel SAR received data by (25) and (26), and obtain the coherence coefficients $\alpha$ and $\gamma$.

Step 2: Through comparing $\alpha$ and $\gamma$, the azimuth sampling scheme is determined. In the case of uniform-sampling or under-sampling, the aliasing number can be directly obtained as $M$; otherwise, $N$ is calculated by (24).

Step 3: Implement azimuth fast Fourier transform (FFT) to each receiver channel data and obtain the multichannel output expressed in range-Doppler domain.

Step 4: Based on the odd-even character of $M$ and the calculated aliasing number $N$, estimate the 


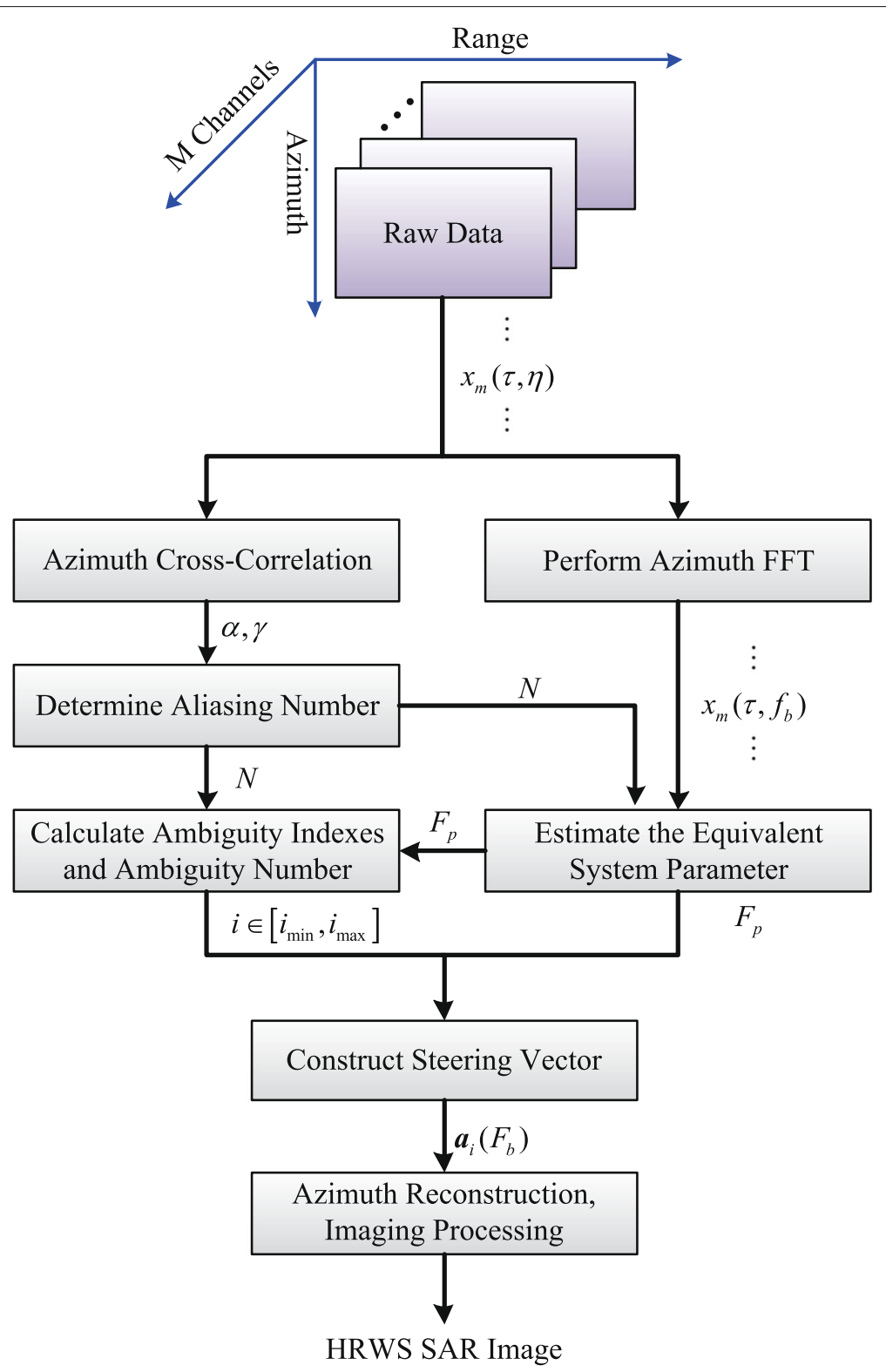

Fig. 4 Algorithm steps of the proposed adaptive azimuth reconstruction method for multichannel HRWS SAR

equivalent parameter $F_{p}$ by the principles of Capon, MUSIC or ESPRIT method.

Step 5: Calculate the ambiguity indexes and ambiguity number of every Doppler bin by (16) or (17).

Step 6: With the ambiguity index $i$ and $F_{p}$, construct the Doppler-variant steering vector $\boldsymbol{a}_{i}\left(F_{b}\right)$.

Step 7: Suppress azimuth ambiguities by MMSE criterion proposed in $[10,20]$ and the idea in [21] to reduce computation load. Finally, obtain the HRWS SAR image by using conventional imaging algorithm, like the chirp scaling (CS) algorithm [1].

We can use several Doppler bins to estimate $F_{p}$ and then average these estimates in order to improve the estimation accuracy. Besides, the assumption that the signal is bandlimited can be relaxed due to the utilization of the MMSE criterion.

\subsection{Computation complexity}

Here, the computation load of the determination of aliasing number $N$ and equivalent system parameter $F_{p}$ is investigated. Since the multiplications need more computation time and hardware sources, we only consider the calculation burden of multiplication. The computation complexity of the proposed adaptive reconstruction method is listed in Table 1. Assume that there are $N_{a}$ azimuth samples in one range gate for each channel data of multichannel SAR, and $K$ Doppler bins are selected to 
Table 1 Computation complexity of our adaptive reconstruction method

\begin{tabular}{lll}
\hline Parameter dimension & Operation & Computation complexity \\
\hline$M, N_{a}, L, K$ & 1.N & $3 \cdot K \cdot M \cdot N_{a}$ \\
& 2. $F_{p}$ & $K \cdot\left(L \cdot M^{2}+\mathcal{O}\left(M^{3}\right)\right)$ \\
& $\hat{\mathbf{R}\left(F_{b}\right)}$ & $L \cdot M^{2}$ \\
& $F_{p}\left(F_{b}\right)$ & $\boldsymbol{O}\left(M^{3}\right)$ \\
& Total & $3 K M N_{a}+K\left(L M^{2}+\mathcal{O}\left(M^{3}\right)\right)$ \\
\hline
\end{tabular}

improve the estimation accuracy of coherence coefficients and the equivalent parameter.

According to Eqs. (19), (20) and (24), the number of complex multiplication for determining the aliasing number $N$ is $3 \cdot K \cdot M \cdot N_{a}$. Based on Eq. (34), the sample covariance matrix $\hat{\boldsymbol{R}}\left(F_{b}\right)$ needs $L \cdot M^{2}$ complex multiplications. In addition, the estimation of $F_{p}\left(F_{b}\right)$ by using spatial spectrum techniques as presented in our paper requires $\mathcal{O}\left(M^{3}\right)$ complex multiplications for each Doppler bin. Thus, the computation complexity for estimating equivalent system parameter is $K \cdot\left(L \cdot M^{2}+\mathcal{O}\left(M^{3}\right)\right)$. Therefore, we can conclude that the total computation complexity for our method is $3 K M N_{a}+K\left(L M^{2}+\mathcal{O}\left(M^{3}\right)\right)$. With the relationship that $N_{a} \gg M, N a \gg K$ and $N a \gg L$, we can confirm that the computation complexity of our method is not high and can be easily implemented.

\section{Experiment results and discussion}

To verify the effectiveness and evaluate the performance of our proposed adaptive azimuth reconstruction method, we conduct extensive experiments on simulated data and real multichannel SAR data. We perform evaluation of the adaptive determination of aliasing number $N$. Then, the validity of the estimated equivalent parameter $F_{p}$ and its performance over SNRs and azimuth sampling uniformity $\kappa$ are demonstrated. We also analyze how the errors of $F_{p}$ estimated by our method affect the azimuth reconstruction. Finally, the real airborne multichannel SAR data is utilized to verify the effectiveness of our reconstruction method.

\subsection{Simulations about aliasing number}

A six-channel SAR system with relevant parameters summarized in Table 2 is employed to generate the simulated data and then implement the following simulation experiments. From the perspective of the aliasing number $N$, we perform comparisons between the proposed adaptive reconstruction method and several other state-of-the-art reconstruction methods including Krieger DBF [3], IDBF [18], and ImpMMSE [20]. Note that the weighting factor $\rho$ is set to be 0.5 in the ImpMMSE reconstruction method. The performance of these aforementioned algorithms over the uniformity of azimuth sampling $\kappa$ would
Table 2 Relevant parameters of the azimuth six-channel SAR system for simulation experiments

\begin{tabular}{lll}
\hline Parameter & Symbol & Value \\
\hline Carrier wavelength & $\lambda$ & $0.059 \mathrm{~m}$ \\
Platform velocity & $v_{r}$ & $7100 \mathrm{~m} / \mathrm{s}$ \\
Slant range & $R_{0}$ & $850 \mathrm{~km}$ \\
Single antenna length & $D$ & $2 \mathrm{~m}$ \\
Optimum PRF & $f_{p o}$ & $1183.3 \mathrm{~Hz}$ \\
Number of channels & $M$ & 6 \\
3dB-Doppler bandwidth & $B_{d}$ & $6290.6 \mathrm{~Hz}$ \\
Range bandwidth & $B_{r}$ & $150 \mathrm{MHz}$ \\
Range sampling frequency & $f_{r}$ & $180 \mathrm{MHz}$ \\
\hline
\end{tabular}

be investigated. The uniformity of azimuth sampling $\kappa$ is set to be from 0.9 to 1.2 , where $\kappa=1$ indicates the uniform-sampling and $\kappa=1.2$ just the coincidingsampling. Moreover, for the sake of comparison, the overall reference is compared to all methods. The values of overall reference are achieved by a single channel SAR with the operational PRF $M f_{p}$. Besides, for the purpose of evaluating AASR, the Doppler bandwidth $B_{D}$ is set to be $8165 \mathrm{~Hz}$ yet the $3 \mathrm{~dB}$-Doppler Bandwidth $B_{d}$ is $6290.6 \mathrm{~Hz}$. Independent white noise is added to the simulated multichannel SAR data such that the SNR of raw data is $20 \mathrm{~dB}$.

Firstly, we conduct experiment with respect to the estimation of coherence coefficients $\alpha$ and $\gamma$. All of the available azimuth samples are used to calculate $\alpha$ and $\gamma$. Figure 5 shows the calculation results of coherence $\alpha$ and $\gamma$ versus $\kappa$. In spite of the varying $\kappa$, the value of $\alpha$ is 0.27 . The value of $\gamma$ rises with the increasing $\kappa$ when $\kappa$ is in the range from 0.9 to 1.2 . We see that the curves $\alpha$ and

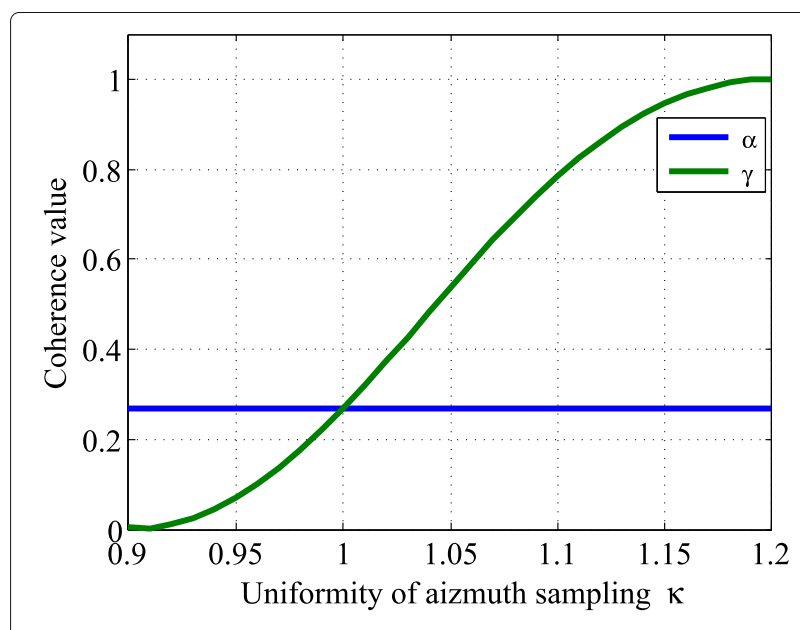

Fig. 5 Calculation result of coherence $\alpha$ and $\gamma$ versus the uniformity of azimuth sampling $\kappa$ 
$\gamma$ intersect at $\kappa=1$ where the azimuth sampling is uniform. Observe that $\alpha>\gamma$ when the azimuth sampling is under-sampling $(0.9 \leq \kappa<1)$ and $\alpha<\gamma$ in case of over-sampling $(1<\kappa \leq 1.2)$. We also observe that $\gamma$ reaches 1 in the case of coinciding-sampling $(\kappa=1)$. The calculation results are in good agreement with theoretical analysis as discussed in Section 4.1. This experiment proves that the estimation of $\alpha$ and $\gamma$ by (25) and (26) have high accuracy. Therefore we can claim that the uncertainty of estimation for coherence values $\alpha$ and $\gamma$ is negligible, which lays a foundation for the evaluation of aliasing number $N$.

Essentially, a primary difference among the above reconstruction methods is the calculation of aliasing number $N$. The aliasing number reflects the reconstructed EDB and hence controls the azimuth resolution. Figure 6

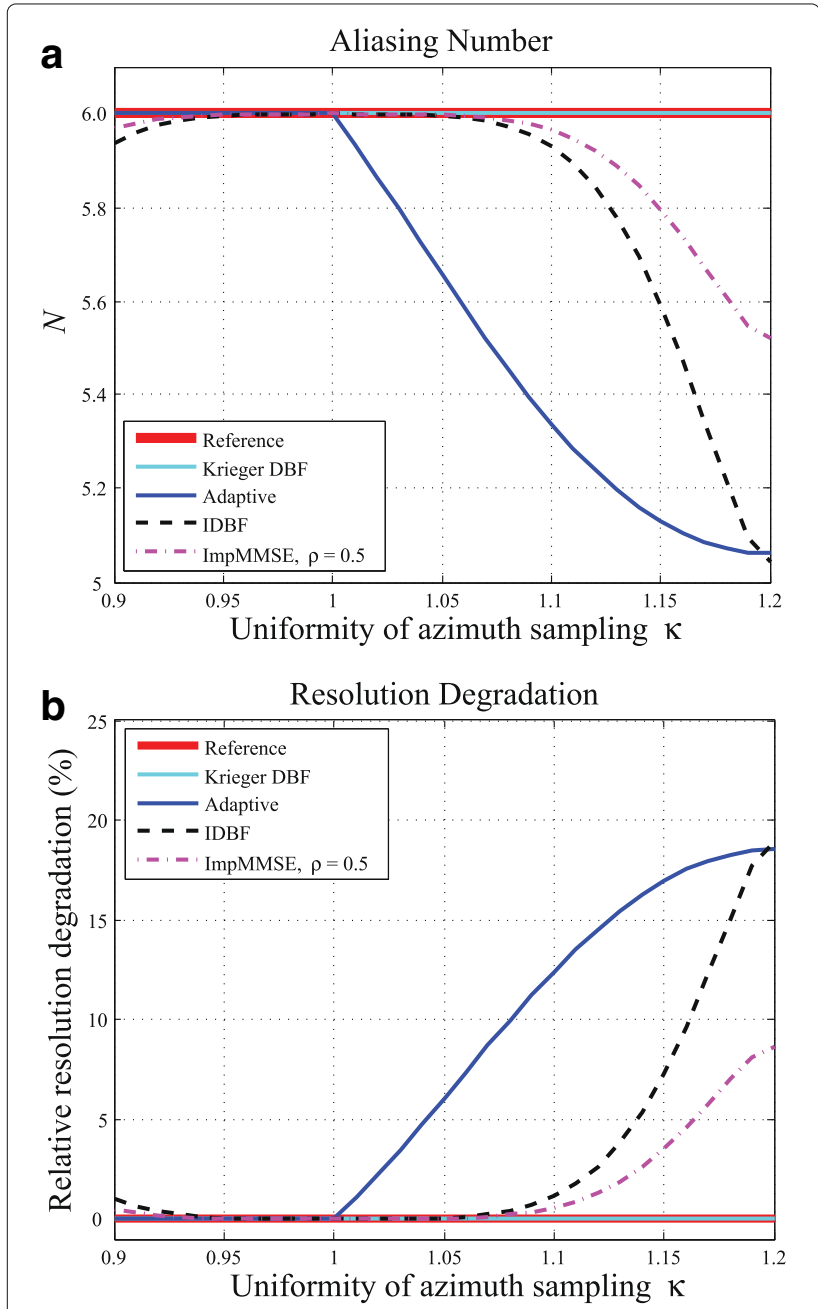

Fig. 6 Aliasing number $N$ and relative resolution degradation. a Aliasing number obtained by different reconstruction methods against the uniformity of azimuth sampling $\kappa$. b Relative resolution degradation obtained by different reconstruction methods against $\kappa$ shows the aliasing number and the relative resolution degradation (in percentage) against varying $\kappa$ realized by different reconstruction methods, respectively. The value of the difference between the resolution and reference resolution divided by the reference resolution is just the relative resolution degradation. From Fig. 6a, we can see that the aliasing number determined by Krieger DBF is constant and equivalent to $M$. In the case of over-sampling $(\kappa>1)$, the aliasing numbers obtained by our method, IDBF and ImpMMSE all reduce with an increasing $\kappa$. The aliasing number of our method decreases more quickly, which infers that its EDB is closer to $M \cdot f_{p o}$. Moreover, we note that the aliasing number of IDBF is slightly inferior to that of our method when $\kappa$ approaches 1.2. One can also notice that the aliasing numbers of IDBF and ImpMMSE are less than $M$ in some under-sampling cases, which violates the fact that the ambiguity number is always $M$ for any Doppler bin $f_{b}$ when $\kappa<1$. Figure 6b shows that
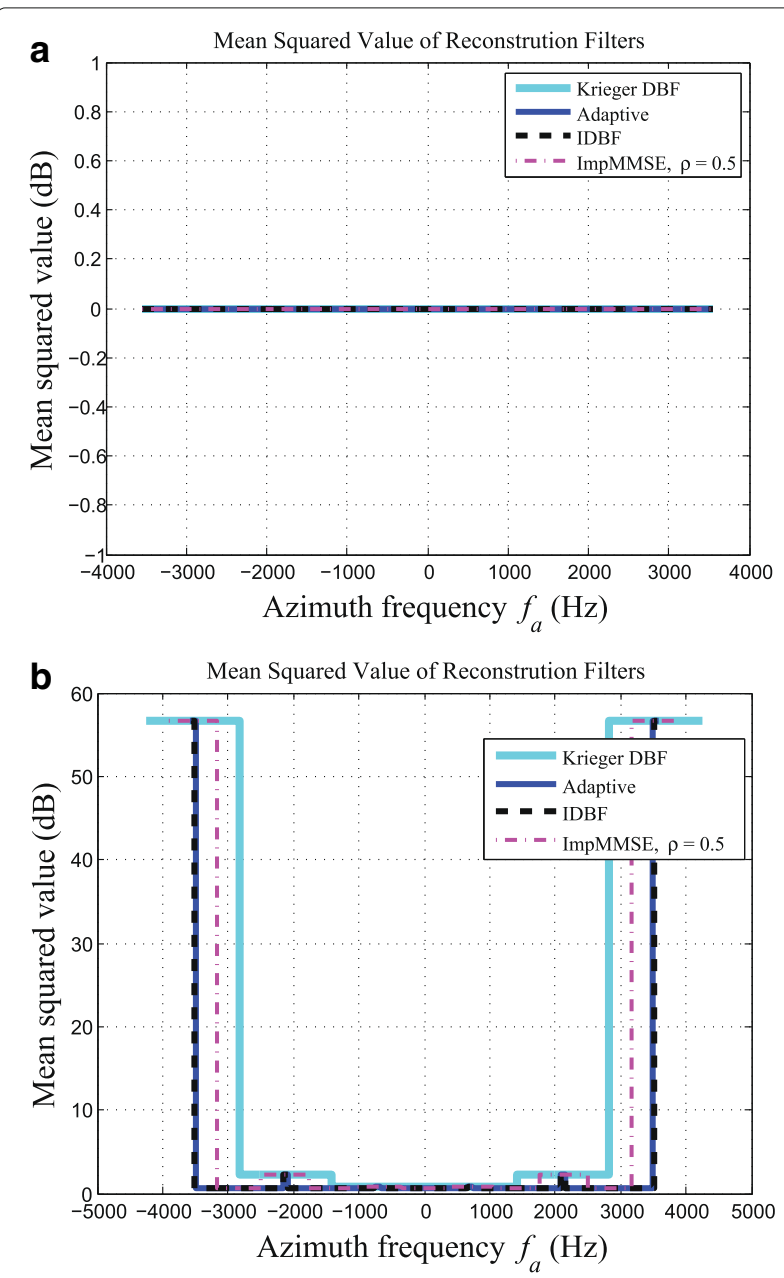

Fig. 7 The mean squared values of reconstruction filters calculated by different reconstruction methods. a The uniform-sampling scheme. b The over-sampling scheme $(\kappa=1.195)$ 
the relative resolution degradations of our method, IDBF and ImpMMSE are all under 20\%, which are maintained at acceptable levels. We know that the azimuth resolution of multichannel SAR is determined by the EDB. The ambiguity suppression in multichannel SAR can achieve excellent performance when EDB reaches $M f_{p o}$. Although our method seems to provide the 'worst' performance in resolution comparing with other methods in case of oversampling, its EDB is closest to $M f_{p o}$. Moreover, the SNR scaling factor $\Phi_{b f}$ and AASR could better represent the reconstruction performance. The finest azimuth resolution does not mean the best performance of ambiguity suppression. Next we will implement simulations to evaluate the reconstruction performance realized by different algorithms.

The curves of mean squared values of reconstruction filter obtained by different methods are plotted in Fig. 7 .
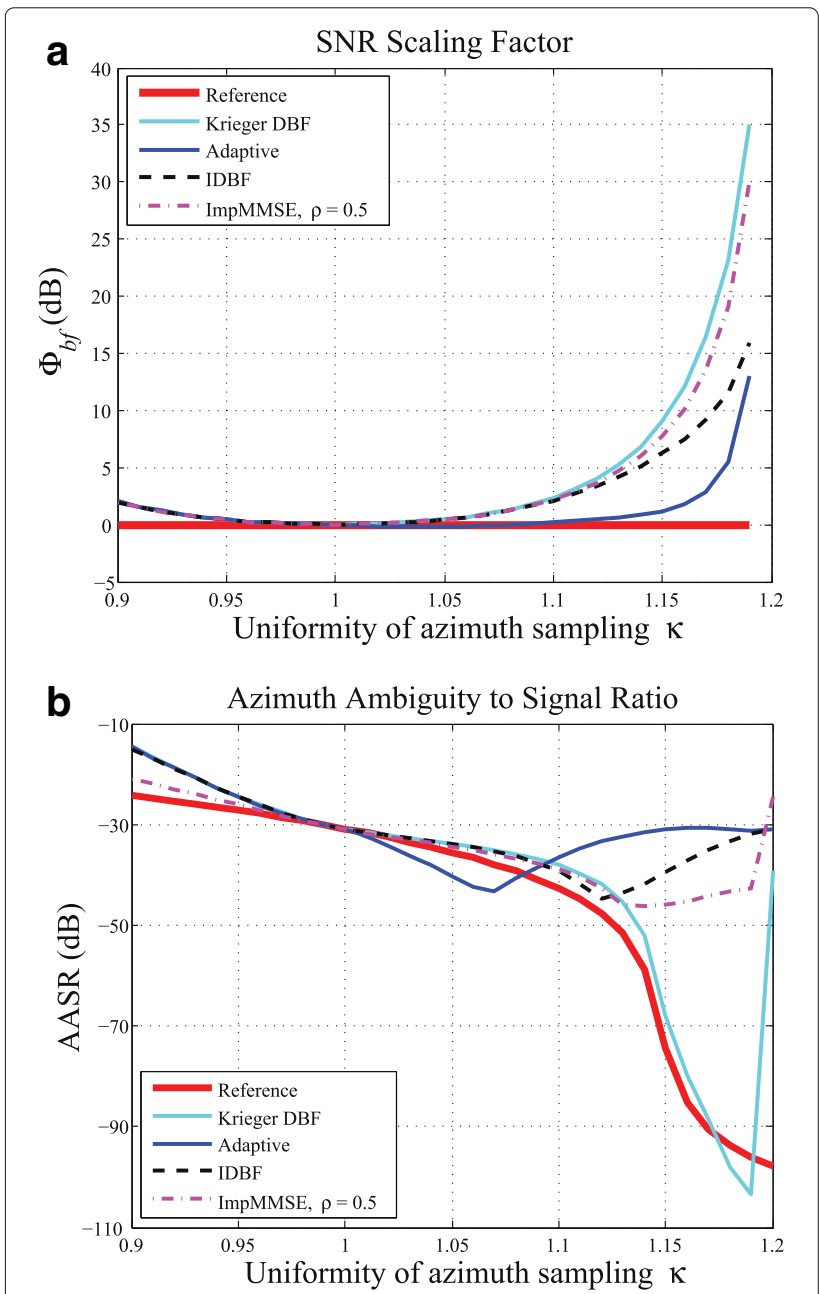

Fig. 8 Reconstruction performance achieved by different algorithms. a The value of SNR scaling factor $\Phi_{b f}$ versus the uniformity of azimuth sampling $\kappa . \mathbf{b}$ The value of AASR versus the uniformity of azimuth sampling $\kappa$
In the uniform-sampling scheme, we observe that the mean values of the reconstruction filter over the instantaneous Doppler frequency $f_{a}$ are all equivalent to $0 \mathrm{~dB}$ (see Fig. 7a), and hence all the four reconstruction methods have similar performance. Whereas, the mean squared values over the outmost Doppler band are much higher in the case of over-sampling as illustrated in Fig. 7b. Bear in mind that the uniformity of the over-sampling case is $\kappa=1.195$, which makes $f_{p}$ approach the operational singular PRF $1414.1 \mathrm{~Hz}$ (generate coinciding-sampling). The rising mean squared values of the over-sampling case can be explained by the increasing condition number of the array manifold $\boldsymbol{A}\left(F_{b}\right)$ [34]. Furthermore, the aliasing number and AIS operation, to a certain extent, are beneficial to reduce the value of condition number of $\boldsymbol{A}\left(F_{b}\right)$. As a consequence, we can see that the IDBF and our reconstruction method have less range of rising value than the ImpMMSE and Krieger DBF method. The rising mean squared value dominates the deterioration of SNR, indicating an increasing SNR scaling factor denoted by $\Phi_{b f}$ [5]. Although the reduction of $N$ can enhance the SNR of the reconstructed azimuth signal, it might result in the drop of AASR [5, 20].

We further study the performance of these algorithms about SNR scaling factor $\Phi_{b f}$ and AASR versus $\kappa(0.9 \leq$ $\kappa \leq 1.2)$. Figure 8a shows the curves of SNR scaling factors obtained by different methods. We note that when $\kappa$ is close to 1 , all methods realize the best performance in terms of $\Phi_{b f}$. Too large deviation from $\kappa=1$ will magnify the SNR scaling factors of all methods, implying the degradation of SNR for the reconstructed signal. Obviously, the proposed method outperforms its competitors regarding SNR scaling factor. This is because our method has the minimum aliasing number (see Fig. 6) and carries

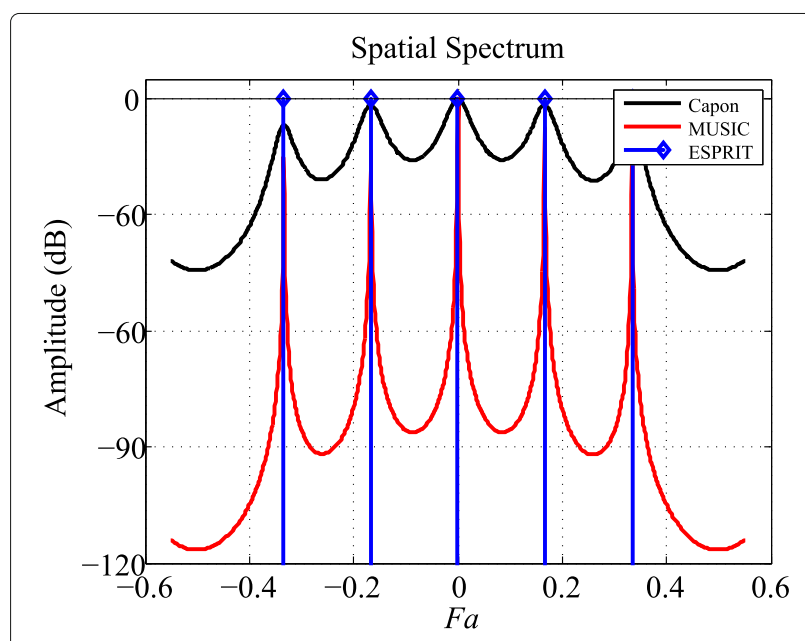

Fig. 9 Envelopes of spatial spectrum realized by different estimation methods for the six-channel SAR simulated data 
out the AIS operation. However, the reduce of aliasing number may degrade the AASR.

Figure $8 \mathrm{~b}$ presents the AASR achieved by different methods. Clearly, when the azimuth sampling is highly over-sampling, the degradation of AASR occurs in our proposed method, the IDBF method and the ImpMMSE method. The proposed method has optimum performance, even better than the reference case, when the uniformity of azimuth sampling $\kappa$ is close to 1 . Although the AASR of our method is slightly inferior to those of other methods in the case of highly over-sampling, its AASR is still under $-30 \mathrm{~dB}$. Considering that there should be a good balance between the SNR and AASR for the reconstructed signal, our adaptive method can be an excellent method to suppress azimuth ambiguities especially for nonuniform sampling cases. Moreover, our method dose not require any system parameters.

\subsection{Simulations on the equivalent system parameter}

We firstly use the simulated data with parameters listed in Table 2 to verify the effectiveness of the adaptive estimation of the equivalent system parameter $F_{p}$, which is
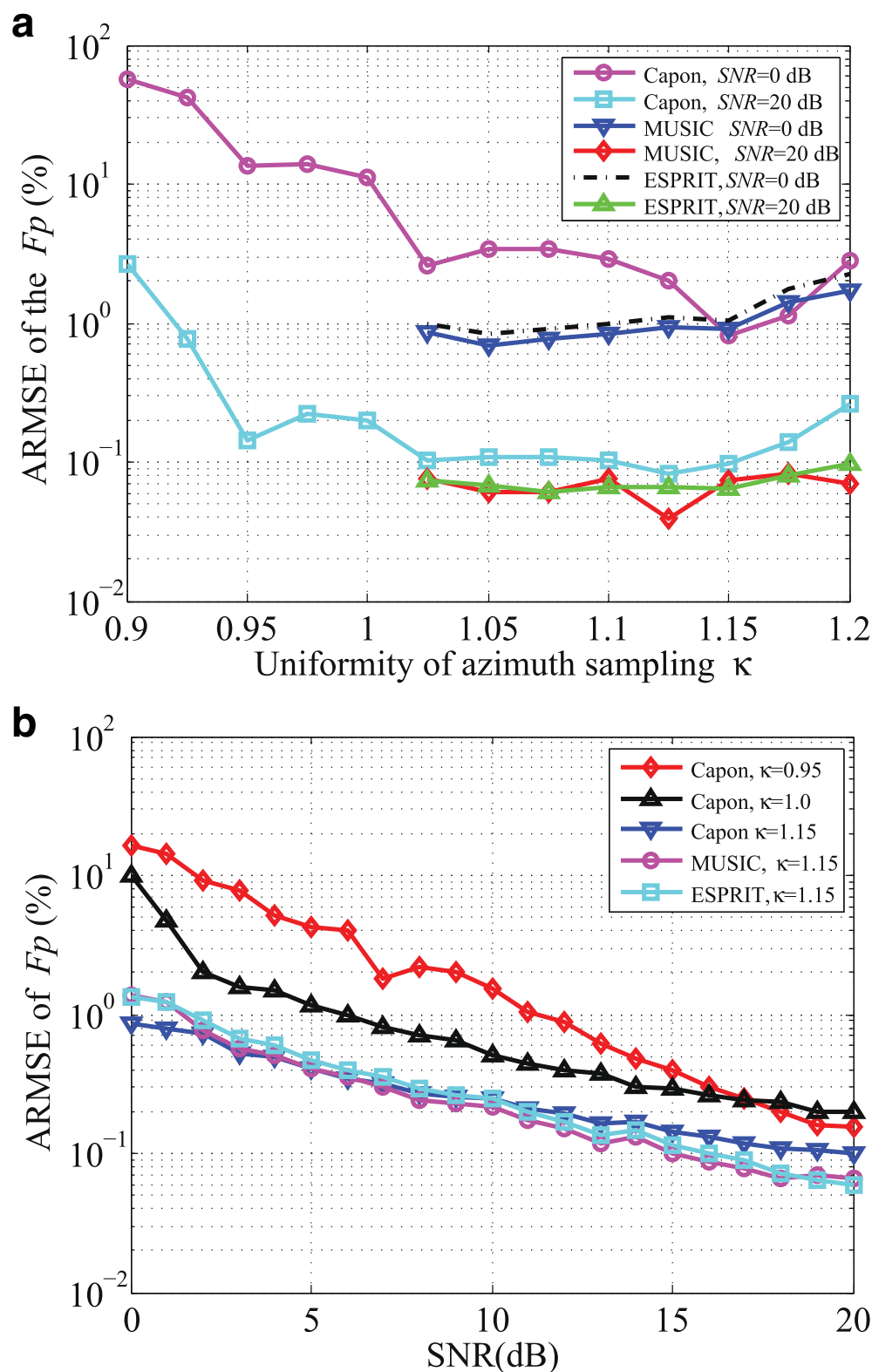

Fig. 10 ARMSE curves of $F_{p}$ by means of different spatial spectrum estimation techniques. a ARMSE versus SNR in different cases of $\boldsymbol{\kappa}$. b ARMSE versus $\kappa$ under conditions with distinct SNRs 
inspired by spatial spectrum estimation techniques. Then we evaluate the estimation accuracy of $F_{p}$ in terms of the average root-mean-squared error (ARMSE) versus SNR and the uniformity of azimuth sampling $\kappa$, respectively. We also investigate how the estimation errors of $F_{p}$ affect the azimuth reconstruction of multichannel SAR signal.

Here the uniformity of azimuth sampling is set to be $\kappa=1.1$ for the convenience of using MUSIC and ESPRIT techniques. Thus, the number of ambiguities in frequency bins around zero Doppler are 5 (cf. (17)). Besides, the range of $F_{a}$ is from -0.55 to 0.55 and the SNR is $20 \mathrm{~dB}$ in this simulation. The spectrum envelopes at zero Doppler bin generated by different estimation techniques are plotted in Fig. 9. Observe that the peaks are uniformly spaced; the peaks of MUSIC and ESPRIT spectrums are sharper than Capon spectrum. The distance between adjacent spectrum peaks just equals the equivalent parameter $F_{p}$. Therefore, we can confirm that $F_{p}$ can be estimated without using any system parameters, which makes our algorithm more practically applicable.

We further investigate the performance of estimating $F_{p}$ versus SNR under conditions with various $\kappa(0.95,1$,
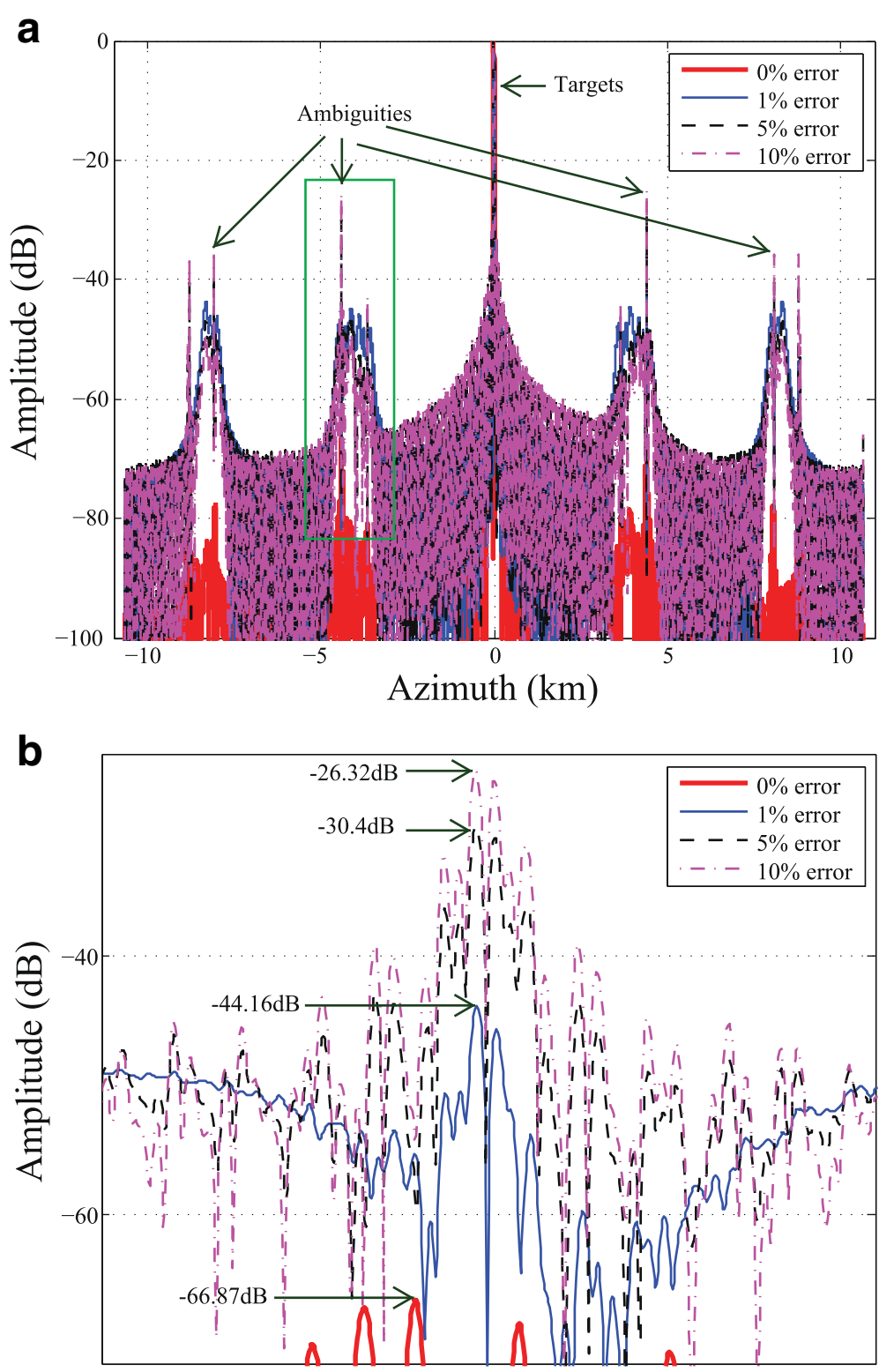

Azimuth $(\mathrm{km})$

Fig. 11 Profiles of the azimuth PSFs for multichannel SAR when there are estimation errors in $F_{p}$. The azimuth PSFs for the point target and ambiguitites; $\mathbf{b}$ Enlarged view of the ambiguities in the green rectangle of $\mathbf{a}$ 
and 1.15). Note that, in the case of $\kappa=1.15$, the three spectrum estimation techniques all can be utilized. Based on 200 trails, the empirical results regarding the ARMSE of $F_{p}$ (in percentage) are shown in Fig. 10a. From an overall perspective, the estimation accuracy is fairly good. One can see that the estimation accuracy improves with the increasing SNR. Compared with the under-sampling scheme, the over-sampling scheme achieves better accuracy. This experiment implies that the estimation accuracy is sensitive to the uniformity of azimuth sampling $\kappa$. Next, we explore the effect of $\kappa$ on the estimation of $F_{p}$.

Figure 10b plots the curves of ARMSE versus $\kappa$ in distinct cases where SNR is 0 and $20 \mathrm{~dB}$, respectively. Notice that the MUSIC and ESPRIT techniques are only utilized in the over-sampling schemes where $\kappa \geq 1.025$. Clearly, the estimation accuracy of $F_{p}$ improves with a rising $\kappa$. Furthermore, we see that the ARMSE achieves the minimum value when $\kappa$ is close to 1.05 , but not 1 . This can be explained that a high PRF is beneficial to enhance the estimation accuracy of $F_{p}$ under condition that the azimuth sampling is quasi-uniform.

We can confirm that in terms of estimating $F_{p}$ the subspace based methods have higher accuracy than Capon method from Fig. 10. The MUSIC method achieves slightly better performance than the ESPRIT method. Whereas, the Capon method and MUSIC method involves the spectral peak searching.

According to Fig. 10, we know that the estimation errors of $F_{p}$ are inevitable. These errors may degrade the performance of ambiguity suppression. Next, we conduct experiments about reconstructing azimuth signal under circumstance that errors are added to $F_{p}$. $F_{p}$ is set to be 1.195 and the aliasing number is adaptively decided by our method. Figure 11a plots the profiles of azimuth point spread functions (PSFs) when different errors $(0,1,5$, and $10 \%$ of the original $F_{p}$ ) are added to the equivalent system parameter $F_{p}$. Figure $11 \mathrm{~b}$ demonstrates the enlargement of the ambiguities located at the green rectangle of Fig. 11a. The PSF results show that the azimuth ambiguities can still be suppressed to $-26.32 \mathrm{~dB}$ even when the error of $F_{p}$ is $10 \%$. Furthermore, in general, the estimation error is considerably inferior to $10 \%$ as illustrated in Fig. 10 . Therefore, we can conclude that our method is effective and efficiency. On the other hand, the deterioration of ambiguity suppression indicates the importance and necessity of the exact system parameters. This experiment also verifies that our reconstruction method obtain good performance in highly nonuniform sampling case.

\subsection{Demonstration with real airborne four-channel SAR data}

Real data collected by an airborne multichannel SAR system is utilized to validate the effectiveness of our adaptive azimuth reconstruction method. The airborne
Table 3 Main parameters of airborne azimuth multichannel SAR system developed by IECAS

\begin{tabular}{lll}
\hline Parameter & Symbol & Value \\
\hline Carrier wavelength & $\lambda$ & $0.03125 \mathrm{~m}$ \\
Platform velocity & $v_{r}$ & $162.6 \mathrm{~m} / \mathrm{s}$ \\
Slant range & $R_{0}$ & $25.6 \mathrm{~km}$ \\
Single antenna length & $D$ & $0.144 \mathrm{~m}$ \\
Optimum PRF & $f_{p o}$ & $564.44 \mathrm{~Hz}$ \\
Operational PRF & $f_{p}$ & $749.76 \mathrm{~Hz}$ \\
Number of channels & $M$ & 4 \\
Range bandwidth & $B_{r}$ & $420 \mathrm{MHz}$ \\
Range sampling frequency & $f_{r}$ & $500 \mathrm{MHz}$ \\
\hline
\end{tabular}

multichannel SAR is developed by the Institute of Electronics, Chinese Academy of Sciences (IECAS) and its main parameters are summarized in Table 3. The channel number of the airborne multichannel SAR system is 4 and hence the optimum PRF $f_{p o}$ is $564.44 \mathrm{~Hz}$. Whereas, the operational PRF of the multichannel SAR is $749.76 \mathrm{~Hz}$ and the uniformity of azimuth sampling $\kappa$ is 1.3283 . Consequently, the multichannel SAR generates a highly nonuniform azimuth sampling considering that $\kappa=$ 1.3333 would lead to the coinciding-sampling scheme. In addition, before the process of azimuth reconstruction, we employ the approach based on the azimuth crosscorrelation to calibrate the unavoidable channel errors $[32,35]$.

The aliasing number $N$ adaptively determined by our method is 3.1542. In contrast, the aliasing number is 3.0242 by IDBF method. When the weighting factor $\rho$ is

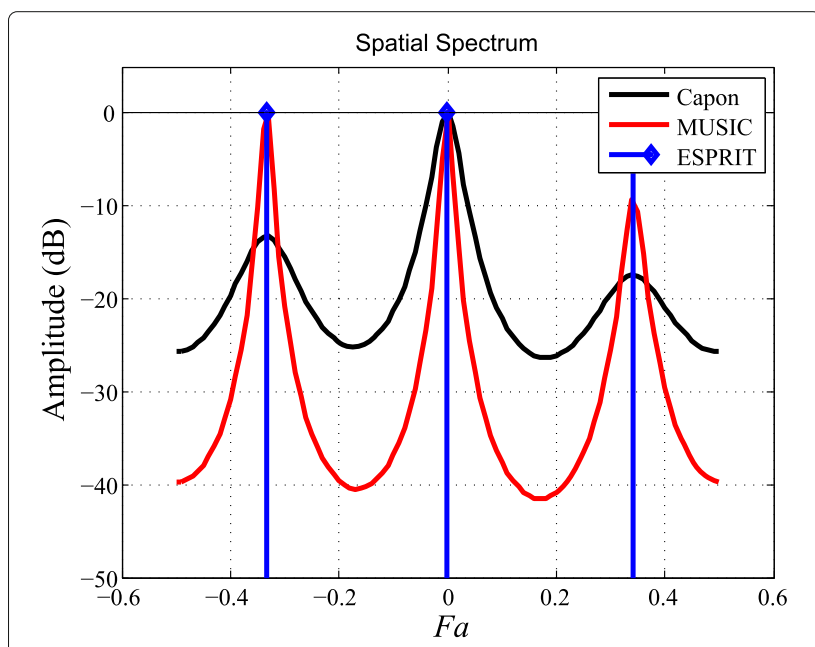

Fig. 12 Envelopes of spatial spectrum achieved by different estimation methods for the real airborne four-multichannel data 
0.5 , the calculated aliasing number is 3.5121 via the ImpMMSE method. Regarding $N$, our method is just equivalent to the ImpMMSE with $\rho=0.8668$ and the IDBF equivalent to the ImpMMSE with $\rho=1$ for the real data. This infers that our method is effective in terms of the calculation of aliasing number and can offer a good tradeoff between AASR and SNR.

Then the equivalent parameter $F_{p}$ is adaptively estimated by our method. Figure 12 plots the spatial spectrums at zero Doppler bin achieved by means of Capon, MUSIC and ESPRIT techniques, respectively. Observe that there are 3 uniformly spaced peaks in every spatial spectrum, which is consistent with the fact that there exist 3 spectrum components at zero Doppler bin. The values of $F_{p}$ calculated from Capon, MUSIC and ESPRIT spectrum are 0.3357, 0.3350, and 0.3355, respectively. Considering that the nominal $F_{p}$ is 0.3321 , the estimation errors for the given $F_{p}$ are 1.0840, 0.8797, and $1.0303 \%$, respectively. With the results shown in Figs. 10 and 11 , we can believe that the estimated $F_{p}$ is fairly accurate, manifesting the efficiency of our method in estimating $F_{p}$.

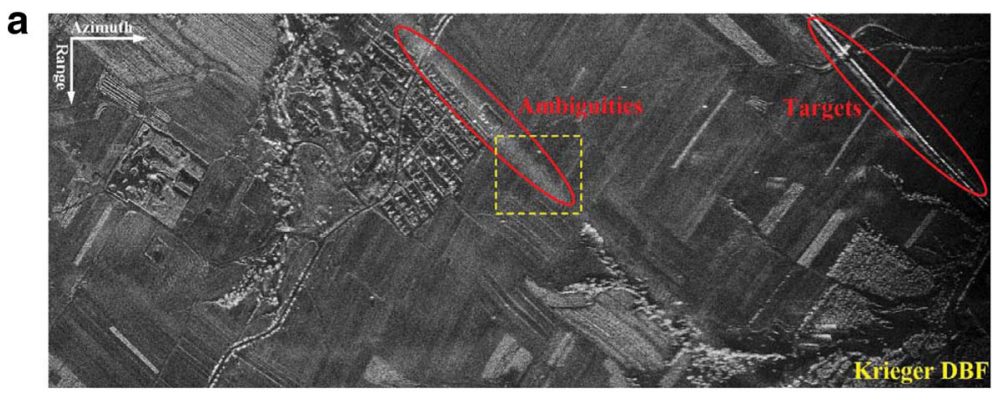

b

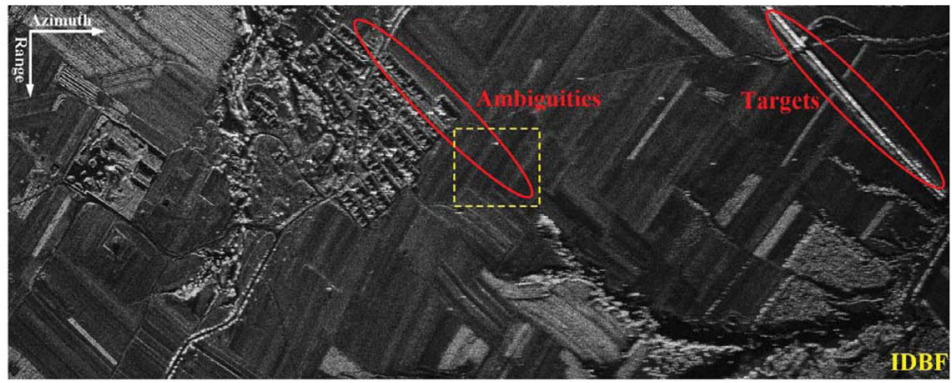

C

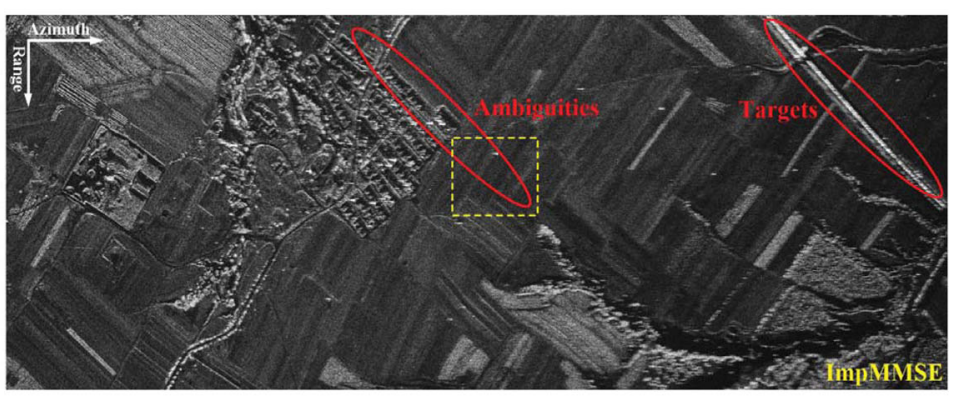

d

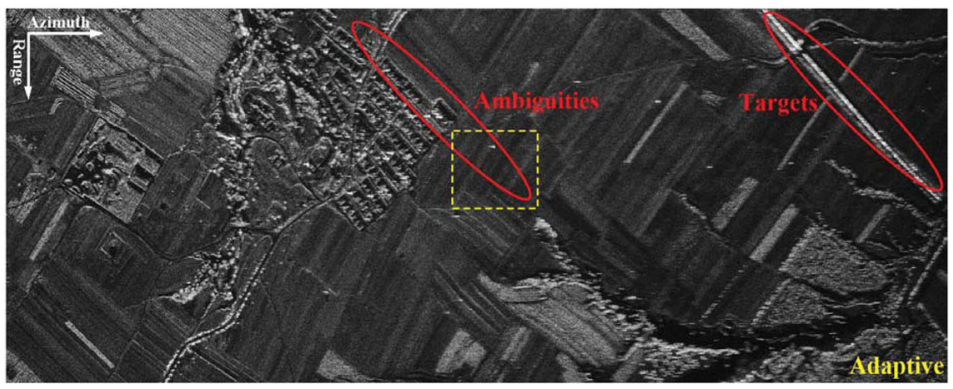

Fig. 13 SAR images using the results reconstructed by different algorithm. a Krieger DBF method. b The IDBF method. c The ImpMMSE method. d Our proposed method 
Afterwards, the azimuth signal is reconstructed by different algorithms, i.e., the Krieger DBF, IDBF, ImpMMSE and the adaptive reconstruction method. It should be noted that our reconstruction method utilizes the adaptively estimated $F_{p}$ via the MUSIC technique while other reconstruction methods employ the given $F_{p}$. With the reconstructed signal, the CS algorithm is employed to perform the imaging procedure [1]. Figure 13 presents the imaging results processed by different reconstruction methods. Observation of the SAR images shows that high level of residual ambiguities are still remained in Fig. 13a which is processed by the Krieger DBF method. In contrast, from Fig. 13b, c, d, we see the azimuth ambiguities can be effectively suppressed by using IDBF, ImpMMSE, and our adaptive reconstruction method, indicating an acceptable balance between the SNR and AASR.

Furthermore, we calculate the relative ambiguity-plusnoise (AN) levels of the yellow dotted rectangles in Fig. 13 to quantitatively evaluate the performance of ambiguity suppression [20]. The area marked by the yellow dotted rectangle is selected for the reason that its image background is relatively pure and weak. In order to make the maximum value of the AN level for the marked zone of Fig. 13a be $0 \mathrm{~dB}$, we normalize the image data achieved by the four methods. To better demonstrate the AN levels, Fig. 14 presents the enlargements of the yellow dotted areas in Fig. 13. The mean AL levels on the marked zone for the Krieger DBF, IDBF, ImpMMSE and our adaptive method are $-31.82,-42.38,-41.23$, and $-44.75 \mathrm{~dB}$, respectively. It can be seen that our proposed adaptive method outperforms its competitors in terms of the real data processing. Therefore we can verify that our adaptive method is a promising candidate to reconstruct the azimuth signal of multichannel SAR even in highly nonuniform sampling scheme. Furthermore, compared with its competitors, another great advantage of our adaptive method is that it does not need any system parameters.

\section{Conclusions}

We have proposed an adaptive azimuth reconstruction algorithm for the multichannel HRWS SAR signal. To this end, we proposed to classify the azimuth sampling of multichannel SAR and derived the calculation formula of ambiguity indexes. Then we presented the two crucial procedures of our adaptive method: the determination of aliasing number $N$ and the estimation of the equivalent system parameter $F_{p}$. Utilizing the azimuth cross-correlation, the aliasing number can be adaptively
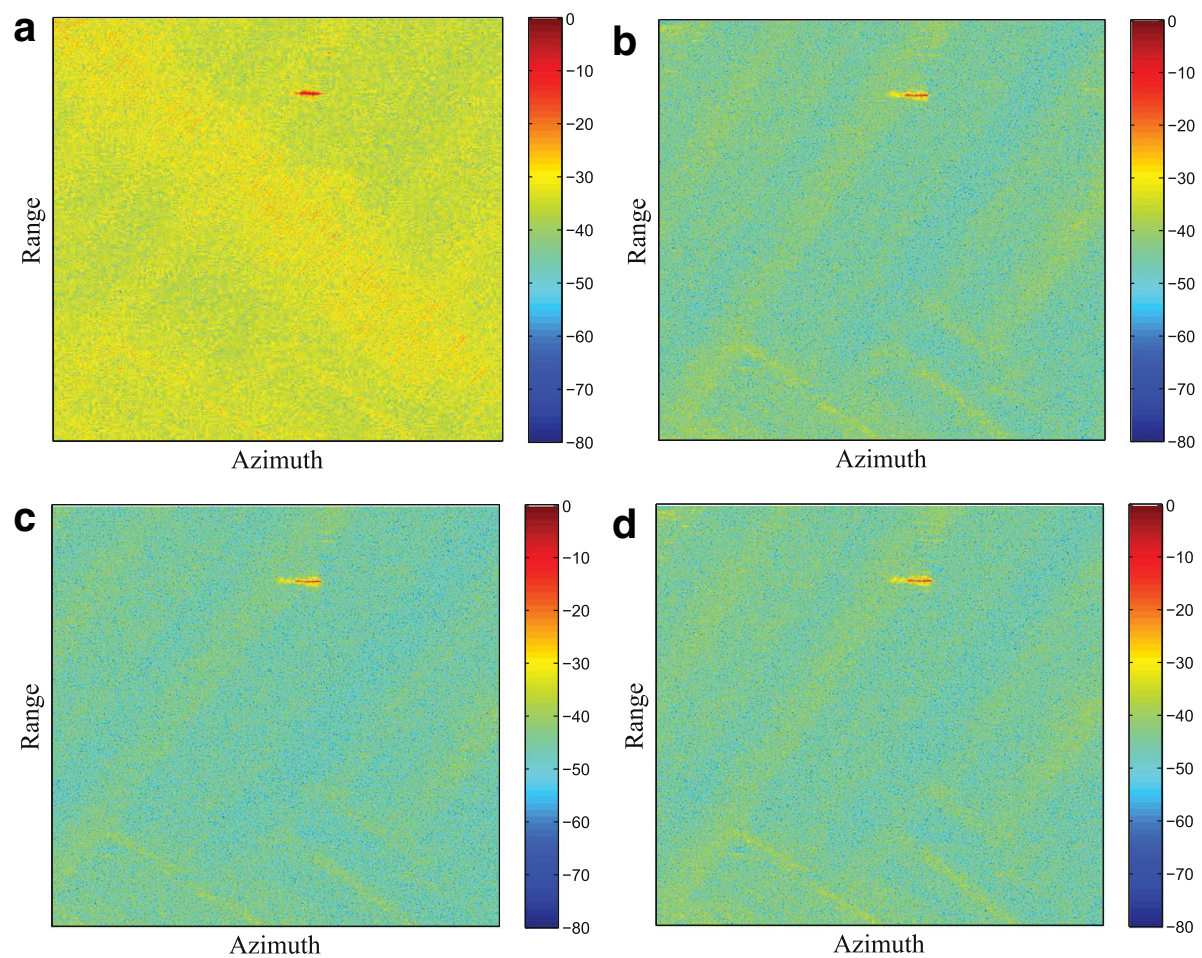

Fig. 14 Enlargements of the yellow dotted zones of Fig. 14. a The ambiguity-plus-noise levels achieved by Krieger DBF. b The ambiguity-plus-noise levels achieved by IDBF. c The ambiguity-plus-noise levels achieved by ImpMMSE. $\mathbf{d}$ The ambiguity-plus-noise levels achieved by our adaptive method 
calculated. The principles of these spatial spectrum estimation techniques are employed to estimate $F_{p}$. Then, with $N$ and $F_{p}$, the ambiguity number and ambiguity indexes of each Doppler bin can be obtained. Consequently, we can adaptively create the steering vectors and then reconstruct the azimuth signal without requiring any system parameters. Comprehensive experiments on simulated data and real data of the airborne multichannel SAR developed by IECAS validate that our adaptive algorithm can effectively suppress azimuth ambiguities and offer an excellent balance between AASR and SNR. Therefore, we can claim that our method is a promising method to reconstruct the azimuth signal of the multichannel HRWS SAR even when the azimuth sampling is highly nonuniform.

\section{Abbreviations}

AASR: Azimuth ambiguity-to-signal ratio; AIS: Ambiguity index screening; AN: Ambiguity-plus-noise; ARMSE: Average root-mean-squared error; CS: Chirp scaling; DBF: Digital beamforming; DSD: Doppler spectrum diagram; EDB: Effective Doppler bandwidth; EPC: Effective phase center; ESPRIT: Estimation of signal parameters via rotational invariance technique; FFT: fast Fourier transform; HRWS: high-resolution and wide-swath; IECAS: Institute of electronics, Chinese academy of sciences; MMSE: Minimum mean square error; MUSIC: Multiple signal classification; PRF: Pulse repetition frequency; Rx: Receiver; SAR: Synthetic aperture radar; SNR: Signal-to-noise ratio; STAP: Space-time adaptive processing; Tx: Transmitter

\section{Acknowledgements}

The authors also thank the anonymous reviewers for their comments and suggestions, which will improve the quality of this manuscript. The authors also thank IECAS for providing the multichannel airborne SAR raw data to this manuscript.

\section{Funding}

This work was supported by the National Natural Science Foundation of China under Grant 61601285.

\section{Authors' contributions}

LZ conceived the main idea, conducted the experiment, discussed the results, and wrote the manuscript. YG and XL supervised the main idea and provided many valuable suggestions to the study. All authors read and approved the final manuscript.

\section{Competing interests}

The authors declare that they have no competing interests.

\section{Publisher's Note}

Springer Nature remains neutral with regard to jurisdictional claims in published maps and institutional affiliations.

Received: 22 July 2017 Accepted: 6 June 2018

Published online: 22 June 2018

\section{References}

1. IG Cumming, FH Wong, Digital Processing of Synthetic Aperture Radar Data: Algorithms and Implementation. (Artech house, Norwood, 2005)

2. A Moreira, P Prats-Iraola, M Younis, G Krieger, I Hajnsek, KP Papathanassiou, A tutorial on synthetic aperture radar. IEEE Geosci. Remote Sens. Mag. 1(1), 6-43 (2012)

3. G Krieger, N Gebert, A Moreira, Unambiguous SAR signal reconstruction from nonuniform displaced phase center sampling. IEEE Geosci. Remote Sens. Lett. 1(4), 260-264 (2004)
4. Z Li, H Wang, T Su, Z Bao, Generation of wide-swath and high-resolution SAR images from multichannel small spaceborne SAR systems. IEEE Geosci. Remote Sens. Lett. 2(1), 82-86 (2005)

5. N Gebert, G Krieger, A Moreira, Digital beamforming on receive techniques and optimization strategies for high-resolution wide-swath SAR imaging. IEEE Trans. Aerosp. Electron. Syst. 45(2), 564-592 (2009)

6. N Gebert, Multi-channel azimuth processing for high-resolution wide-swath SAR imaging. PhD thesis. Ger. Aerosp. Center., 1-224 (2009)

7. QXu, G Liao, A Liu, J Zhang, Self-calibration method without joint iteration for distributed small satellite SAR systems. EURASIP J. Adv. Signal Process. 2013(31), 1-10 (2013)

8. H Yang, J Yang, Z Li, J Wu, Y Huang, L Teng, Near-space slow SAR high-resolution wide swath and sustained imaging technology. EURASIP J. Wirel. Commun. Netw. 2013(57), 1-8 (2013)

9. D Cerutti-Maori, I Sikaneta, J Klare, C H. Gierull, MIMO SAR processing for multichannel high-resolution wide-swath radars. IEEE Trans. Geosci. Remote Sens. 52(8), 5034-5055 (2014)

10. I Sikaneta, D Cerutti-Maori, J Klare, Optimum signal processing for multichannel SAR: With application to high-resolution wide-swath imaging. IEEE Trans. Geosci. Remote Sens. 52(10), 6095-6109 (2014)

11. J Tang, Y Deng, R Wang, S Zhao, N Li, W Wang, A weighted backprojection algorithm for azimuth multichannel SAR imaging. IEEE Geosci. Remote Sens. Lett. 13(9), 1265-1269 (2016)

12. L Zhang, Y Gao, X Liu, A robust channel phase error calibration algorithm for multichannel HRWS SAR imaging. IEEE Geosci. Remote Sens. Lett. 14(5), 649-653 (2017)

13. T Yang, Z Li, Z Suo, Y Liu, Z Bao, Performance analysis for multichannel HRWS SAR systems based on STAP approach. IEEE Geosci. Remote Sens. Lett. 10(6), 1409-1413 (2013)

14. J Capon, High-resolution frequency-wavenumber spectrum analysis. Process. IEEE. 57(8), 1408-1418 (1969)

15. YC Eldar, AV Oppenheim, Filterbank reconstruction of bandlimited signals from nonuniform and generalized samples. IEEE Trans. Signal Process. 48(10), 2864-2875 (2000)

16. E Margolis, YC Eldar, Nonuniform sampling of periodic bandlimited signals. IEEE Trans. Signal Process. 56(7), 2728-2745 (2008)

17. L Zhang, N Zhang, Y Gao, K Wang, X Liu, in 2016 IEEE International Geoscience and Remote Sensing Symposium (IGARSS). Reconstruction of azimuth signal for multichannel HRWS SAR imaging based on periodic extension (IEEE, 2016), pp. 2102-2105

18. B Liu, Y He, Improved DBF algorithm for multichannel high-resolution wide-swath SAR. IEEE Trans. Geosci. Remote Sens. 54(2), 1209-1225 (2016)

19. S-S Zuo, M Xing, X-G Xia, G-C Sun, Improved signal reconstruction algorithm for multichannel SAR based on the Doppler spectrum estimation. IEEE J. Sel. Topics Appl. Earth Observ. Remote Sens. 10(4), 1425-1442 (2017)

20. N Liu, R Wang, Y Deng, S Zhao, X Wang, Modified multichannel reconstruction mmethod of SAR with highly nonuniform spatial sampling. IEEE J. Sel. Topics Appl. Earth Observ. Remote Sens. 10(2), 617-627 (2017)

21. X Guo, Y Gao, K Wang, X Liu, Modified reconstruction algorithm based on space-time adaptive processing for multichannel synthetic aperture radar systems in azimuth. J. Appl. Remote Sens. 10(3), 035022 (2016)

22. L Zhang, Y Gao, K Wang, X Liu, in 2017 IEEE Radar Conference. A blind reconstruction of azimuth signal for multichannel HRWS SAR system (IEEE, 2017)

23. S-X Zhang, M-D Xing, X-G Xia, L Zhang, R Guo, Y Liao, Z Bao, Multichannel HRWS SAR imaging based on range-variant channel calibration and multi-Doppler-direction restriction ambiguity suppression. IEEE Trans. Geosci. Remote Sens. 52(7), 4306-4327 (2014)

24. HA Zebker, J Villasenor, Decorrelation in interferometric radar echoes. IEEE Trans. Geosci. Remote Sens. 30(5), 950-959 (1992)

25. Y-K Kong, B-L Cho, Y-S Kim, Ambiguity-free Doppler centroid estimation technique for airborne SAR using the Radon transform. IEEE Trans. Geosci. Remote Sens. 43(4), 715-721 (2005)

26. PA Rosen, S Hensley, IR Joughin, FK Li, SN Madsen, E Rodriguez, RM Goldstein, Synthetic aperture radar interferometry. Proc. IEEE. 88(3), 333-382 (2000)

27. Z Wang, J Li, R Wu, Time-delay-and time-reversal-based robust capon beamformers for ultrasound imaging. IEEE Trans. Med. Imaging. 24(10), 1308-1322 (2005)

28. D Ciuonzo, G Romano, R Solimene, Performance analysis of time-reversal music. IEEE Trans. Signal Process. 63(10), 2650-2662 (2015) 
29. D Ciuonzo, PS Rossi, Noncolocated time-reversal music: High-snr distribution of null spectrum. IEEE Signal Process. Lett. 24(4), 397-401 (2017)

30. R Schmidt, Multiple emitter location and signal parameter estimation IEEE Trans. Antenn. Propag. 4(3), 276-280 (1986)

31. R Roy, T Kailath, ESPRIT-estimation of signal parameters via rotational invariance techniques. IEEE Trans. Acoust. Speech Signal Process. 37(7), 984-995 (1989)

32. Y Liu, Z Li, Z Wang, Z Bao, On the baseband Doppler centroid estimation for multichannel HRWS SAR imaging. IEEE Geosci. Remote Sens. Lett. 11(12), 2050-2054 (2014)

33. P Stoica, RL Moses, Spectral Analysis of Signals. (Pearson Prentice Hall, Upper Saddle River, NJ, 2005)

34. GH Golub, CF Van Loan, Matrix Computations. 4th edn. (The Johns Hopkins Univers. Press, Baltimore, Maryland, 2012)

35. J Feng, C Gao, Y Zhang, R Wang, Phase mismatch calibration of the multichannel SAR based on azimuth cross correlation. IEEE Geosci. Remote Sens. Lett. 10(4), 903-907 (2013)

\section{Submit your manuscript to a SpringerOpen ${ }^{\circ}$ journal and benefit from:}

- Convenient online submission

- Rigorous peer review

- Open access: articles freely available online

- High visibility within the field

- Retaining the copyright to your article

Submit your next manuscript at $>$ springeropen.com 